\title{
1. Liquid Structure of a Water-in-Salt Electrolyte with a Remarkably Asymmetric Anion
}

\author{
3 Alessandro Triolo, * Valerio Di Lisio, Fabrizio Lo Celso, Giovanni B. Appetecchi, Barbara Fazio, \\ ${ }_{4}$ Philip Chater, Andrea Martinelli, Fabio Sciubba, and Olga Russina*
}

Cite This: https://doi.org/10.1021/acs.jpcb.1c06759

Read Online

\section{ACCESS}

山ll Metrics \& More

回 Article Recommendations

S1 Supporting Information

5 ABSTRACT: Water-in-salt systems, i.e., super-concentrated aqueous electrolytes, such as 6 lithium bis(trifluoromethanesulfonyl)imide $\left(21 \mathrm{~mol} / \mathrm{kg}_{\text {water }}\right)$, have been recently discovered 7 to exhibit unexpectedly large electrochemical windows and high lithium transference 8 numbers, thus paving the way to safe and sustainable charge storage devices. The peculiar 9 transport features in these electrolytes are influenced by their intrinsically nanoseparated 10 morphology, stemming from the anion hydrophobic nature and manifesting as 11 nanosegregation between anions and water domains. The underlying mechanism behind 12 this structure-dynamics correlation is, however, still a matter of strong debate. Here, we 13 enhance the apolar nature of the anions, exploring the properties of the aqueous 14 electrolytes of lithium salts with a strongly asymmetric anion, namely, 15 (trifluoromethylsulfonyl)(nonafluorobutylsulfonyl) imide. Using a synergy of experimental 16 and computational tools, we detect a remarkable level of structural heterogeneity at a 17 mesoscopic level between anion-rich and water-rich domains. Such a ubiquitous sponge-

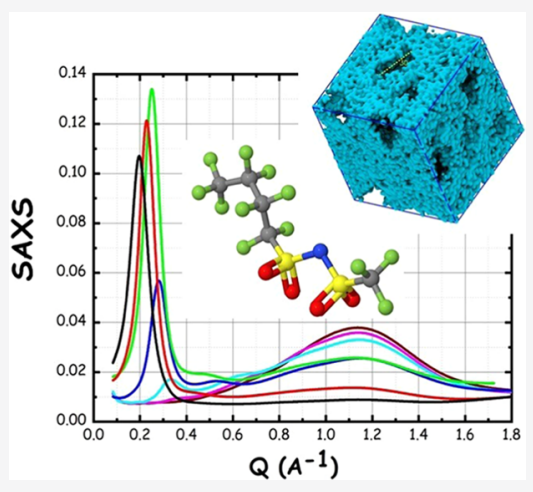
18 like, bicontinuous morphology develops across the whole concentration range, evolving from large fluorinated globules at high 19 dilution to a percolating fluorous matrix intercalated by water nanowires at super-concentrated regimes. Even at extremely 20 concentrated conditions, a large population of fully hydrated lithium ions, with no anion coordination, is detected. One can then 21 derive that the concomitant coexistence of (i) a mesoscopically segregated structure and (ii) fully hydrated lithium clusters 22 disentangled from anion coordination enables the peculiar lithium diffusion features that characterize water-in-salt systems.

23

24 Super-concentrated aqueous electrolytes are presently the 25 focus of intense research since the first experimental data 26 appeared to reveal the unexpected performances of water27 depleted salt solutions in the field of energy storage. ${ }^{1}$ Such 28 systems are nowadays conventionally indicated as water-in-salt 29 (WiS) mixtures to highlight the specific component ratio that 30 characterizes them; in particular, one typically identifies 31 aqueous electrolytes in such a way, when the salt to water 32 ratio is larger than one, both by weight and volume. The 33 interest in these systems stems from the enhanced and rather 34 unexpected electrochemical stability of water-containing 35 electrolytes in the specific concentration regime where WiS 36 are defined. The narrow electrochemical stability of water $37(1.23 \mathrm{~V})$ has traditionally limited aqueous electrolytes from 38 application in energy storage devices. On the other hand, the 39 use of more electrochemically stable organic media to support 40 charge conduction in batteries is prone to potentially severe 41 side effects related to solvent flammability and chemical 42 stability. Accordingly, the discovery by Suo and co-workers 43 that super-concentrated aqueous lithium bis44 (trifluoromethanesulfonyl)imide (LiTFSI) mixtures would 45 perform an electrochemical stability up to ca. $3 \mathrm{~V}$ paved the 46 way to a series of investigations aiming to rationalize and exploit this novel observation. ${ }^{2-16}$ Nowadays, however, several 47 issues remain unexplored with respect to the morphology and 48 the conduction mechanisms taking place in these unconven- 49 tional media. Aqueous electrolytes have been studied in the 50 past in dilute conditions, focusing on solvent-separated ion 51 pairs, where water efficiently fully solvates the ionic species. 52 Upon increasing the salt content, conductivity reaches a 53 maximum and, due to increased viscosity, progressively 54 decreases when the salt content reaches concentrations of 55 the order of a few meters $\left(\mathrm{mol}_{\text {salt }} / \mathrm{kg}_{\text {solvent }}\right)$. Accordingly, the 56 highly concentrated regime that characterizes WiS systems has 57 barely been explored in the past and only recently, more 58 systematic studies are being developed in this new regime. ${ }^{17-36} 59$ Recent reviews have addressed the nature of the structural, 60 dynamic, and electrochemical properties of these sys- 61 tems. $^{2,3,8,15,37-42}$ Much of the structural investigations have 62 been focused on the first WiS system, namely, LiTFSI- $\mathrm{H}_{2} \mathrm{O}, 63$

Received: July 30, 2021

Revised: October 25, 2021 
64 mostly due to LiTFSI high solubility in water $(>20 \mathrm{~m}$ at 25 $65{ }^{\circ} \mathrm{C}$ ) and stability against hydrolysis. ${ }^{1}$ The phase diagram of this 66 binary system has been characterized, ${ }^{18}$ showing the existence 67 of a eutectic LiTFSI $/ \mathrm{H}_{2} \mathrm{O}=1: 1$, with a melting point at ca. $68-40{ }^{\circ} \mathrm{C}$. This behavior has been recently framed in a more 69 general trend involving other unconventional deep eutectic 70 solvents formed by aqueous salt hydrates. ${ }^{43}$ At ca. $21 \mathrm{~m}$ (salt 71 molar fraction $=0.275)$, the mixture has a melting point of 25 $72{ }^{\circ} \mathrm{C}$, representing the system with the highest $\mathrm{Li}$ content, which 73 remains liquid at ambient conditions. LiTFSI was proposed as 74 an electrolyte for aqueous lithium-ion batteries by Lux et al., ${ }^{44}$ 75 and its high concentration mixtures $(c>15 \mathrm{~m})$ show 76 interesting conductivity performances $(5-10 \mathrm{mS} / \mathrm{cm})^{18}$ and 77 an appreciable electrochemical stability, at least up to $2 \mathrm{~V}{ }^{1}$ 78 Nowadays, different options alternative to the LiTFSI- $\mathrm{H}_{2} \mathrm{O}$ $79 \mathrm{WiS}$ are being considered to enhance the resulting perform80 ance, including exploring $\mathrm{Na}$ - and $\mathrm{K}-$ based $\mathrm{WiS}^{2,5,7,8,14,20,45-47}$ 81 or exploiting asymmetric anions and anion mixtures (leading to 82 the so-called water in bisalt systems). 6,9,12,16,24,45,48,49

83 Here, we explore an aqueous electrolyte system with a salt 84 that is characterized by a remarkably asymmetric anion, i.e., a 85 lithium salt with the anion being a member of the family of 86 di(perfluoroalkyl-sulfonyl)imide, namely (trifluoromethylsul87 fonyl) (nonafluorobutylsulfonyl)imide (hereinafter indicated 88 as [IM14]) (see Scheme 1). The high asymmetry of this anion

\section{Scheme 1. Chemical Structure of Lithium \\ (Nonafluorobutanesulfonyl(trifluoromethanesulfonyl)imide $\left(\right.$ LiIM14) ${ }^{a}$}

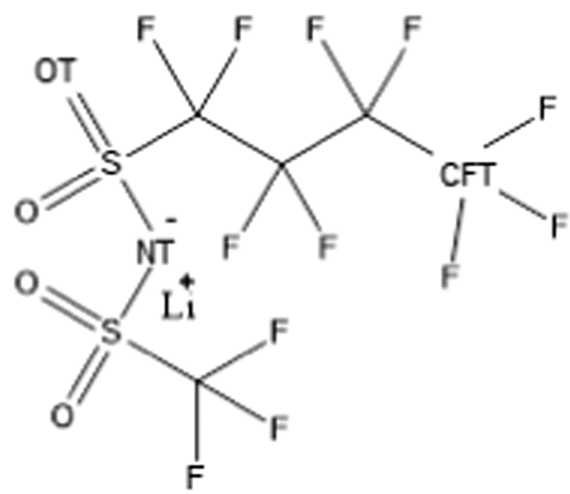

${ }^{a}$ In the discussion of molecular dynamics simulation results, the anion's oxygen, nitrogen, and terminal butyl carbon atoms are identified as OT, NT, and CFT, respectively.

89 makes it an ideal species to pair with cations that are prone to 90 crystallization when paired with more conventional anions. In 91 fact, we recently explored a range of ionic liquid compounds 92 based on imidazolium or other cations paired with the [IM14] 93 anion, highlighting their high tendency to remain in the liquid 94 state even at very low temperatures. ${ }^{50-54}$ Very recently, a 95 manuscript reported the role of anion size in the nanostructure 96 of WiS systems, comparing the morphology detected in 97 LiTFSI-based WiS with that in Li trifluoro-methanesulfonate 98 ( $\mathrm{TfO}$ ) ones, highlighting the importance of the salt volume 99 fraction in influencing the morphology. ${ }^{48}$ In this respect, the 100 present choice for the anion represents an upper limit to the 101 WiS systems studied so far.

102 In this contribution, we will show that LiIM14-based 103 electrolytes are characterized by interesting properties from 104 the point of view of the phase diagram (and hence the liquid state window) and electrochemistry (leading to super- 105 concentrated electrolytes with appealing conductivity and 106 electrochemical stability performances). We will further 107 probe the structural organization in these electrolytes by 108 exploiting the synergy between X-ray scattering, Raman and IR 109 spectroscopies, calorimetry, electrochemical characterization, 110 and molecular dynamics (MD) simulations to provide a robust 111 characterization of the microscopic and mesoscopic organ- 112 ization in these systems. Due to the long perfluoro chain of the 113 anion, a complex mesoscopic morphology develops, as 114 detected by X-ray scattering techniques. The atomistic level 115 description will be obtained by comparison between structural 116 and spectroscopic information with MD results, providing a 117 clear description of the role played by anion hydrophobicity in 118 determining a nanoseparated morphology and of the enduring 119 presence of fully hydrated lithium ions with no anions 120 coordinating them, even at the most concentrated conditions. 121 Despite the importance of chaotropic anions such as IM14 in 122 enabling efficient WiS systems to be developed, ${ }^{40}$ so far, very 123 little structural information exists on the organization of WiS 124 based on salts different from LiTFSI. ${ }^{28,42,55}$ This work aims at 125 expanding the spectrum of available salts that can be envisaged 126 as electrochemically appealing WiS candidates, providing a 127 novel insight into the structural role of long fluorous tails of the 128 anions in affecting the ubiquitous structural heterogeneities in 129 these systems.

130

\section{EXPERIMENTAL AND COMPUTATIONAL DETAILS 131}

Chemicals. The lithium (trifluoromethylsulfonyl)- 132 (nonafluorobutylsulfonyl)imide, LiIM14, salt (see Scheme 1) 133 was synthesized by reacting acidic (trifluoromethylsulfonyl)- 134 (nonafluorobutylsulfonyl)imide (HIM14, $3 \mathrm{M}, 60$ wt \% 135 solution in water) with lithium carbonate $\left(\mathrm{Li}_{2} \mathrm{CO}_{3}\right.$, Fluka, 136 $>99.5$ wt \%) in slight excess ( 2 wt \%) with respect to the 137 stoichiometric amount for pushing the yield up to $100 \%, 138$ according to eq 1 . Both the reagents were used as received. 139

$$
\begin{aligned}
& 2 \mathrm{HIM14}_{\text {(aqueous) }}+\mathrm{Li}_{2} \mathrm{CO}_{3} \rightarrow \mathrm{H}_{2} \mathrm{CO}_{3}+2 \mathrm{Li} \mathrm{IM14}_{\text {(aqueous) }} \\
& \downarrow \\
& \mathrm{H}_{2} \mathrm{O}+\mathrm{CO}_{2} \uparrow
\end{aligned}
$$

Lithium carbonate (solid) was slowly added, as the acid- 141 base reaction is rather exothermal, to avoid excessive heat 142 release. The so-obtained aqueous solution was stirred at room 143 temperature for $30 \mathrm{~min}$ to promote $\mathrm{CO}_{2}$ removal, thus driving 144 the reaction to completeness. Then, the water was removed in 145 a rotary evaporator at $80{ }^{\circ} \mathrm{C}$ for 3-4 h, obtaining a solid, white 146 LiIM14 salt. The $\mathrm{Li}_{2} \mathrm{CO}_{3}$ excess (within LiIM14) was removed 147 by dissolving (stirring at room temperature) the salt in the 148 minimal amount of absolute ethanol (VWR Chemicals, 100 wt 149 $\%)$. Lithium carbonate, insoluble in ethanol, was separated by 150 vacuum filtration (oil-free pump). Successively, the alcoholic 151 LiIM14 solution was subjected to vacuum distillation $\left(50{ }^{\circ} \mathrm{C} 152\right.$ for $2 \mathrm{~h}$ ) to remove ethanol. Finally, the LiIM14 salt was 153 vacuum-dried at $120^{\circ} \mathrm{C}$ overnight to reduce the water content 154 below 5 ppm.

The LiIM14 solutions were prepared by dissolving the 156 proper amount of salt in deionized (Millipore deionizer) water 157 to obtain samples having a molality ranging from 1 to $20 \mathrm{~m}$. 158 The dissolution of LiIM14 in the most concentrated samples 159 (i.e., 15 and $20 \mathrm{~m}$ ) was promoted by stirring at $40-50{ }^{\circ} \mathrm{C}$ for 160 
$16120-30 \mathrm{~min}$. The mixtures were kept in sealed vials until ready 162 for measurements.

163 Differential Scanning Calorimetry (DSC). DSC thermo164 grams were acquired by a Mettler Toledo DSC 822e equipped 165 with an FRS5 sensor and a liquid nitrogen cooler. The furnace 166 was purged during the measurement with dry nitrogen at a 167 flow rate of $30 \mathrm{~mL} / \mathrm{min}$. The samples of about $5 \mathrm{mg}$ were 168 weighed in a $40 \mu \mathrm{L}$ aluminum pan and rapidly sealed. DSC 169 scans comprised of cooling from 50 to $-125^{\circ} \mathrm{C}$ followed by 170 heating from -125 up to $50{ }^{\circ} \mathrm{C}$, with a heating/cooling rate of $1712 / 10{ }^{\circ} \mathrm{C} / \mathrm{min}$.

172 Density. Density data were obtained using a DM45 Mettler 173 Toledo densimeter equipped with a vibrating tube with a 174 resolution of $10^{-5} \mathrm{~g} / \mathrm{cc}$. Measurements were taken as a 175 function of temperature that was controlled to be within $10^{-3}$ $176{ }^{\circ} \mathrm{C}$ by means of a Peltier module. The instrument was 177 calibrated with dry air and degassed-distilled water before 178 performing the experiments.

179 Wide-Angle X-ray Scattering (WAXS). The total high180 resolution X-ray scattering data were collected on the I15-1 181 beamline at Diamond Light Source, U.K., using X-rays of a 182 wavelength of $0.309574 \AA$ and a Perkin Elmer XRD 4343 CT 183 detector. Such a setup allowed covering a $Q$ range between 1840.25 and $20 \AA^{-1}$. The total scattering data were integrated to $1851 \mathrm{D}$ using $\mathrm{DAWN}^{56}$ and then normalized and corrected to 186 extract $I(Q)$. The X-ray structure factors, $S(Q)$, are normalized 187 for the single atomic scattering, according to

$$
S(Q)=\frac{I(Q)-\sum n_{i} f_{i}^{2}(Q)}{\left(\sum n_{i}^{2} f_{i}^{2}(Q)\right)}
$$

188 where $n_{i}$ and $f_{i}(Q)$ are the number concentration and the 189 atomic scattering factors of the $i$ th atomic species. The 190 corresponding quantities are evaluated using molecular 191 dynamics simulations for comparison purposes.

192 The samples were loaded into glue-sealed borosilicate 193 capillaries of a $1.0 \mathrm{~mm}$ outer diameter; measurements were 194 conducted at ambient conditions (ca. $20^{\circ} \mathrm{C}$ ). Additional data 195 were collected at a Bruker D8 Advance diffractometer 196 equipped with a Mo $\mathrm{K} \alpha \mathrm{X}$-ray tube $(\lambda=0.7107 \AA)$, using 197 samples contained in $1.5 \mathrm{~mm}$ diameter quartz capillaries. In 198 this case, the accessible angular range allowed covering 199 between 0.6 and $15 \AA^{-1}$.

200 Small-Angle X-ray Scattering (SAXS). Small-angle X-ray 201 scattering (SAXS) measurements were performed at the 202 SAXSLab Sapienza with a Xeuss 2.0 Q-Xoom system (Xenocs 203 SA, Sassenage, France), equipped with a micro-focus Genix 3D $204 \mathrm{X}$-ray source $(\lambda=0.1542 \mathrm{~nm})$, a two-dimensional Pilatus3 R $205300 \mathrm{~K}$ detector, which can be placed at a variable distance from 206 the sample. Calibration of the scattering vector $Q$ range, where $207 Q=(4 \pi \sin \theta) / \lambda$ and $2 \theta$ is the scattering angle, was performed 208 using a silver behenate standard.

209 Measurements with different sample-detector distances 210 were performed so that the overall explored $Q$ region was $2110.1<Q<3 \AA^{-1}$. The samples were loaded into a disposable 212 quartz capillary with a nominal thickness of $1.0 \mathrm{~mm}$ and sealed 213 with hot glue before placing them in the instrument sample 214 chamber at reduced pressure $(\sim 0.2 \mathrm{mbar})$. The beam size was 215 defined through the two-pinhole collimation system equipped 216 with scatterless slits to be $0.25 \mathrm{~mm} \times 0.25 \mathrm{~mm}$.

217 The two-dimensional scattering patterns were subtracted for 218 the dark counting and then masked, azimuthally averaged, and normalized for transmitted beam intensity, exposure time, and 219 subtended solid angle per pixel using FoxTrot software 220 developed at SOLEIL. The one-dimensional $I(Q)$ vs $Q$ profiles 221 were then subtracted for the capillary contribution. 222

The measurements were conducted at ambient temperature 223 (ca. $20{ }^{\circ} \mathrm{C}$ ), and the samples remained liquid and 224 homogeneous during the whole length of the experiment. 225

Electrochemical Properties. The ion transport properties 226 of aqueous LiIM14 concentrated electrolytes were studied in 227 terms of ionic conductivity vs temperature dependence. The 228 measurements were performed at the temperature ranging 229 from -40 to $80{ }^{\circ} \mathrm{C}$ at a very slow heating scan rate $\left(1{ }^{\circ} \mathrm{C} / \mathrm{h}\right) 230$ for better evidencing the phase transitions. A conductivity- 231 meter AMEL 160, allowing to run impedance measurements at 232 a fixed frequency (i.e., $1 \mathrm{~Hz}$ or $1 \mathrm{kHz}$, depending on the 233 conduction value of the sample under test), was used, whereas 234 the temperature control was performed using a climatic test 235 chamber (Binder GmbH MK53). The electrolytes were 236 housed in sealed glass conductivity cells (AMEL 192/K1) 237 equipped with two porous platinum electrodes. The cell 238 constant (depending on the geometric characteristics of the 239 cell under test, $\sim 1.00 \mathrm{~cm}^{-1}$ ) was previously determined 240 through a $0.1 \mathrm{~N} \mathrm{KCl}$ aqueous solution having an exactly known 241 conductivity value. Typical uncertainties on the conduction 242 data are within 5\%. The error bar in the conductivity plot (vide 243 infra) falls within the data markers. To fully crystallize the 244 aqueous LiIM14 electrolytes, the cells were dipped in liquid 245 nitrogen for $60 \mathrm{~s}$ and then immediately transferred into the 246 climatic chamber (previously set-up at $-40{ }^{\circ} \mathrm{C}$ ). This route 247 was repeated until the frozen electrolytes remained solid at 248 $-40{ }^{\circ} \mathrm{C}$. Finally, the cells were kept at $-40{ }^{\circ} \mathrm{C}$ for at least $24 \mathrm{~h} 249$ prior to starting the conductivity measurements. The 250 reproducibility of the conductivity data was verified by running 251 the measurement set 2 times (from -40 to $80{ }^{\circ} \mathrm{C}$ ) described 252 above.

253

The anodic stability (toward oxidation) was evaluated by 254 linear sweep voltammetry (LSV) carried out on a symmetrical, 255 platinum, two-electrode (thickness and diameter equal to 100256 $\mu \mathrm{m}$ and $10 \mathrm{~mm}$, respectively) cells. The Pt electrodes, 257 sandwiching a glass fiber separator (10 mm diameter), were 258 housed within T-shape poly(propylene) containers, using steel 259 rods (10 $\mathrm{mm}$ diameter) as the current collectors. The 260 electrolytes under test (about $1 \mathrm{~mL}$ ) were loaded into the 261 cell containers, which were then locked to avoid liquid leakage. 262 The measurements were carried out at $1 \mathrm{mV} / \mathrm{s}$ and room 263 temperature, using a PAR 2273 galvanostat/potentiostat, by 264 scanning the cell voltage from the OCV value toward more 265 positive (anodic limit) voltages. Clean electrodes and fresh 266 cells were used for each test. To confirm the reproducibility of 267 the results, the LSV tests were run at least twice on different 268 fresh cells.

269

Raman and Infrared Spectroscopy. Raman spectra were 270 acquired at room temperature using a LabRam HR800 Raman 271 Spectrometer (Horiba Jobin Yvon), equipped with an 272 Olympus BX41-microscope accessorized for macro investiga- 273 tion (a $4 \times$ magnification objective and a multipass cell holder). 274 The $\mathrm{He}-\mathrm{Ne}$ laser beam at $\lambda=632.8 \mathrm{~nm}$ was focused with a 275 power of $3 \mathrm{~mW}$ on the sample placed in a glass cuvette. The 276 Raman scattered light was collected in a backscattering 277 configuration via the same illumination objective, dispersed 278 by a $600 \mathrm{~L} / \mathrm{mm}$ grating, and then detected through a Peltier- 279 cooled silicon CCD (Synapse by Horiba Jobin Yvon). The 280 spectra were typically acquired with integration times of 120 s. 281 
282 Infrared spectra of LiIM14-water mixtures were acquired at 283 room temperature and in the transmission mode using a 284 Nicolet FTIR 6700 Spectrometer by Thermo Fisher Scientific. 285 Solutions were pressed between two $\mathrm{ZnSe}$ windows $(2 \mathrm{~mm}$ 286 thickness) without using a spacer to avoid signal saturation and 287 held in position by a liquid cell holder purchased from Specac. 288 Spectra were recorded in the $4000-400 \mathrm{~cm}^{-1}$ range by co289 adding 100 scans at a resolution of $2 \mathrm{~cm}^{-1}$.

290 MCR-ALS Spectral Decomposition. FTIR and Raman 291 spectra in the water absorption region were analyzed using a 292 Multivariate Curve Resolution-constrained Alternating Least 293 Squares (MCR-ALS) bilinear prediction model ${ }^{57,58}$ (mcr_tool294 box 2 add-on) implemented in MATLAB software. Using the 295 MCR-ALS model, Infrared and Raman spectra of LiIM14 296 solutions were decomposed into a linear combination of 297 several absorbing species contributing to the concentration298 dependent spectral variation. Two data sets were created and 299 analyzed separately, the first containing FTIR absorbance 300 spectra in a spectral range composed of the $4000-2850 \mathrm{~cm}^{-1}$ $301 \mathrm{OH}$ stretching region and the $1850-1450 \mathrm{~cm}^{-1} \mathrm{HOH}$ bending 302 region. A second dataset was formed by the Raman intensities 303 spectra in the $\mathrm{OH}$ stretching region $\left(4000-2800 \mathrm{~cm}^{-1}\right)$. The 304 MCR-ALS algorithm decomposed an initial dataset in a 305 product of two smaller matrices, the first containing the 306 spectral profiles of the absorbing species and the second 307 comprised of the concentration profiles (or spectral 308 coefficients). First, an MCR-ALS model comprising three 309 spectral components was chosen by performing a preliminary 310 principal component analysis. This model accounts for 99.7 311 and $99.8 \%$ of the total spectral variance for the FTIR and 312 Raman data sets, respectively. The initial estimation of the 313 three spectral profiles was performed by singular value 314 decomposition (SVD). Finally, the convergence of the iterative 315 fitting procedure was achieved when the standard deviation fell 316 below 0.0001 for both FTIR and Raman data sets. To obtain 317 meaningful information, a non-negative constraint was applied 318 to both concentration and spectral profiles, and closure 319 constraint was applied to keep the sum of the concentration 320 profiles equal to $1 .^{59}$ Final fittings were characterized by a LOF $321 \%$ (lack of fit) parameter of $2.3 \%$ for FTIR and $2.7 \%$ for Raman 322 data sets.

323 Molecular Dynamics (MD) Simulations. Classical MD 324 simulations for LiIM14 WiS were performed at different 325 concentrations consistent with experimental data sets. In 326 particular, we simulated systems with $c=1,2,5,7,10,15$, and $32720 \mathrm{~m}$ for the LiIM14- $\mathrm{H}_{2} \mathrm{O}$ system.

328 MD simulations were performed using GROMACS 2018.3 329 package software ${ }^{60,61}$ Bonded and nonbonded parameters for 330 the IM14 anion were described using an all-atoms 331 potential; $^{62-64}$ the SPCE water model was used for the 332 solvent. $^{65}$ The Li-ion potential was taken from ref 66 .

333 The simulations for LiIM14-water solutions were per334 formed using cubic boxes; the initial edge size was fixed 335 between 8.5 and $10 \mathrm{~nm}$ depending on the concentration; 336 periodic boundary conditions were applied. We stress that 337 large simulation boxes were required to satisfactorily reproduce 338 the experimentally determined structural properties, and more 339 conventional smaller boxes would have missed to grasp 340 fundamental structural features. The initial configurations 341 were created by Packmol software. ${ }^{67}$ The equilibration 342 procedure was performed in several steps, starting from an 343 NVT simulation at $400 \mathrm{~K}$ and scaled partial charges (10\% of 344 the original ones), followed by a series of NPT runs lowering the temperature progressively (from 400 to $350 \mathrm{~K}$ ) and 345 increasing the charges to their final value ( $80 \%$ of the original 346 ones) at $298 \mathrm{~K}$ and 1 bar after a $6 \mathrm{~ns}$ run. After the 347 equilibration phase, each system was run for at least $150 \mathrm{~ns}$ for 348 the production run, and then a further trajectory of $4 \mathrm{~ns}$ was 349 saved at a frequency of 2 ps for the calculation of structural 350 properties. The production simulations were always checked vs 351 the energy profile. During the production runs for the 352 temperature coupling, we used a velocity rescaling thermo- 353 stat $^{68}$ (with a time coupling constant of $0.1 \mathrm{ps}$ ), while for the 354 pressure coupling, we used a Parrinello-Rahman barostat ${ }^{69}$ (1 355 ps for the relaxation constant). The leap-frog algorithm with a 356 $1 \mathrm{fs}$ time step was used for integrating the equations of motion. 357 Cut-offs for the Lennard-Jones and real space part of the 358 Coulombic interactions were set to $15 \AA$ A. For the electrostatic 359 interactions, the particle mesh Ewald (PME) summation 360 method $^{70,71}$ was used, with an interpolation order of 6 and 361 $0.08 \mathrm{~nm}$ of FFT grid spacing. Selected graphs were done using 362 VMD. ${ }^{72}$ Weighted and partial structure factors were computed 363 using in-house developed software, while the selected pair 364 correlation function and angular distribution function were 365 obtained by TRAVIS. ${ }^{73-75}$ Analysis of the shortest contiguous 366 hydrogen-bond path between every pair of water molecules as 367 well as the path between hydrogen-bonded water molecules 368 and water molecules connected via lithium interaction has 369 been conducted using ChemNetworks software. ${ }^{76}$

\section{RESULTS AND DISCUSSION}

All of the solutions probed in the present study are 372 thermodynamically stable in their liquid state above $25{ }^{\circ} \mathrm{C} .373$ The LiIM14-water electrolytes are herein reported for the first 374 time, and their phase diagram has not been published so far. 375 Figure S-1 shows the DSC traces for LiIM14- $\mathrm{H}_{2} \mathrm{O}$ mixtures, 376 in the concentration range between 1 and $20 \mathrm{~m}$, at a heating 377 rate of $2{ }^{\circ} \mathrm{C} / \mathrm{min}$. This information leads to a proposal for the 378 phase diagram of the LiIM14-water system over the presently 379 reported concentration range (see Figure 1). We mention $380 \mathrm{fl}$ herein that both WiS with LiIM14 at $c=15$ and $20 \mathrm{~m}$, when 381 cooled from the melt, remained in the liquid state at 382 temperatures around $20{ }^{\circ} \mathrm{C}$, where some measurements were 383 conducted, despite their melting point being slightly above this 384 value: accordingly, we likely characterized a slightly super- 385

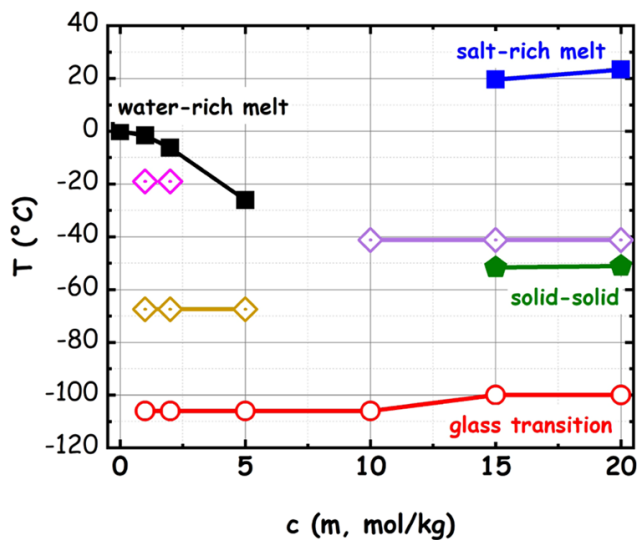

Figure 1. Phase diagram of the LiIM14- $\mathrm{H}_{2} \mathrm{O}$ system obtained from calorimetric measurements. Full symbols refer to strong endothermic transitions; open symbols refer to the liquid-glass transition; dotted symbols refer to weak, spike-like features. Lines are guides for the eye. 

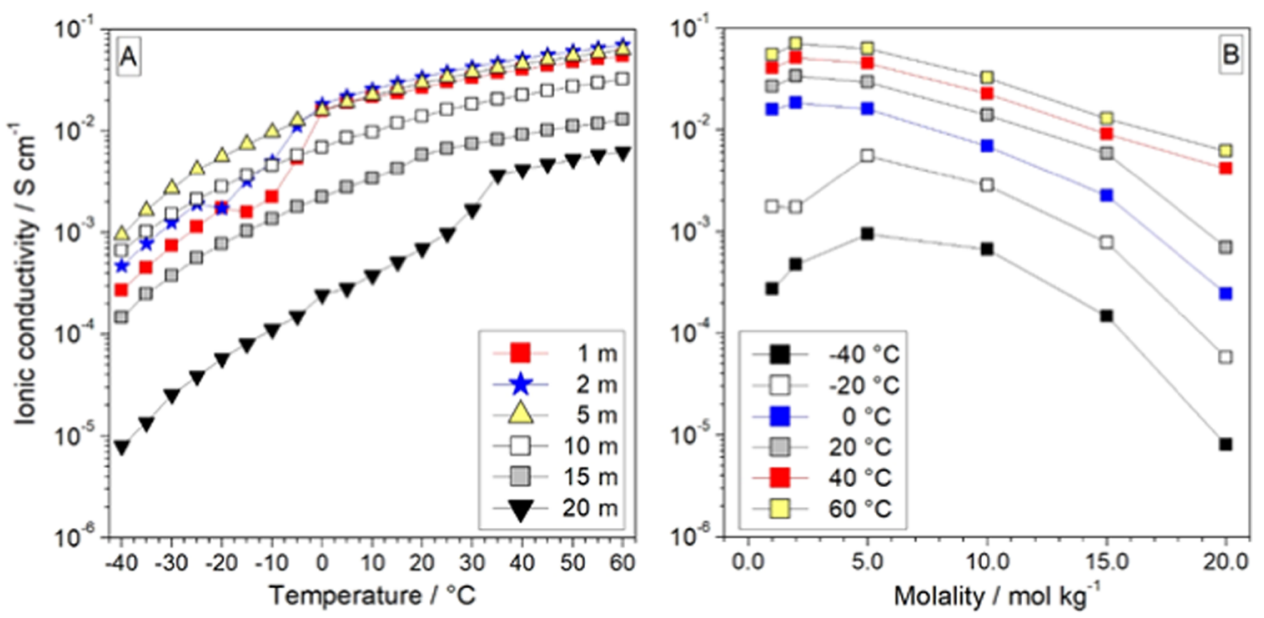

Figure 2. (a) Ionic conductivity of the LiIM14- $\mathrm{H}_{2} \mathrm{O}$ system as a function of temperature for different salt contents; (b) different isotherms for the ionic conductivity of the LiIM14- $\mathrm{H}_{2} \mathrm{O}$ system, as a function of salt content.

386 cooled state of these mixtures in our experimental X-ray 387 studies. The LiTFSI-water phase diagram is highly related to 388 the presently reported LiIM14-water one. Ding and $\mathrm{Xu}^{18}$ 389 reported the whole phase diagram for aqueous LiTFSI for $0 \leq$ $390 x_{\text {LiTFS }} \leq 1$, at ambient pressure. For this study, they used 391 samples containing carbon microbeads to facilitate nucleation 392 events that might lead to a safer characterization of solid 393 phases. In our present study, we do not use such an option: 394 accordingly, some crystallization events might have been 395 overlooked. LiTFSI $-\mathrm{H}_{2} \mathrm{O}$ features an articulated phase 396 diagram, and two different hydrates have been detected 397 therein: namely, LiTFSI $\cdot\left(\mathrm{H}_{2} \mathrm{O}\right)_{4}$ and LiTFSI $\cdot\left(\mathrm{H}_{2} \mathrm{O}\right)$ that 398 have been assumed to be formed by positively charged 399 hydrated lithium ions paired with the anion. Our present study 400 does not allow extracting this information due to the limited 401 number of samples considered. We notice that upon increasing 402 the salt content in neat water, a progressive decrease of the 403 water-rich $(c \leq 5 \mathrm{~m})$ mixture melting point is observed. These 404 mixtures are characterized by two further solid-solid 405 transitions at -19 and $-67.5{ }^{\circ} \mathrm{C}$ that appear as very weak 406 calorimetric features (presumably due to incomplete trans407 formations) and, eventually, at low enough temperature, by a 408 glass transition event at $-106{ }^{\circ} \mathrm{C}$ (without appreciable 409 concentration dependence). At more concentrated conditions $410(c=10 \mathrm{~m})$, only a very tiny feature is observed at $-41.2^{\circ} \mathrm{C}$, 411 and otherwise, the sample is subjected to a liquid-glass 412 transition at $-106{ }^{\circ} \mathrm{C}$. This specific concentration looks 413 peculiar, as no strong endothermic events seem to occur. It 414 corresponds to an $x_{\text {LiIM14 }}=0.2$ salt molar fraction and seems 415 relatively easy to be supercooled to the amorphous state, 416 without intervening crystallization, seemingly leading to a 417 eutectic composition (this might provide a hint to the 418 existence of stable hydrates with stoichiometry: LiIM14. $419\left(\mathrm{H}_{2} \mathrm{O}\right)_{4}$; further research is active on this topic). A higher 420 salt content leads to a shift of glass transition toward higher 421 temperature (i.e., a more rigid environment), and a cold 422 crystallization and subsequent melting can be observed at 423 higher temperatures. Eventually, a solid-liquid transition 424 occurs at approximately room temperature. These systems 425 will need to be further investigated with greater detail; 426 nevertheless, valuable information on the liquid state 427 conditions and on the existence of several crystalline phases 428 can be safely assessed.
The temperature dependence of the ionic conductivity for 429 selected LiIM14-water electrolytes is reported in Figure 2a. $430 \mathrm{f} 2$ Analogous behavior and high reproducible conduction values 431 were obtained from two measurement sets, indicating good 432 reliability of the results. All investigated electrolyte samples, 433 with the exception of the $20 \mathrm{~m}$ one, show conductivity values 434 ranging from $10^{-4}$ to $10^{-3} \mathrm{~S} / \mathrm{cm}$, i.e., of interest for practical 435 applications, already at $-40{ }^{\circ} \mathrm{C}$. This experimental evidence, 436 supporting a gained ion mobility in the frozen state likely due 437 to the very large steric hindrance of the IM14 anion, makes the 438 LiIM14 WiS solutions appealing for electrochemical devices 439 operating at very low temperatures. The conductivity behavior 440 of the $c=1$ and $2 \mathrm{~m}$ samples in the range between -25 and 441 $-15{ }^{\circ} \mathrm{C}$ might confirm the existence of solid-solid phase 442 transitions and/or different ion rearrangement prior to the 443 melting temperature in this concentration range at ca. $-19{ }^{\circ} \mathrm{C} 444$ (Figure S-1), in which the ions show lower mobility.

445

Conversely, no evident conductivity jump is detected for the 446 $c=5$ and $10 \mathrm{~m}$ samples within the whole investigated 447 temperature range. The more concentrated electrolytes $(c=15448$ and $20 \mathrm{~m}$ ) exhibit a conductivity increase from $-40{ }^{\circ} \mathrm{C}$ up to 449 room temperature, likely ascribable to the progressive 450 structural reorganization of ions and/or solid-solid phase 451 transitions. Around 20 and $30{ }^{\circ} \mathrm{C}$ increase in moderate 452 conductivity (particularly for the $c=15 \mathrm{~m}$ sample) is observed, 453 indicating melting of the $c=15$ and $20 \mathrm{~m}$ electrolytes, 454 consistently with calorimetric results. In the molten state, the 455 ionic conductivity of the LiIM14- $\mathrm{H}_{2} \mathrm{O}$ solutions exhibits a 456 Vogel-Fulcher-Tammann trend, which displays, as expected, 457 a progressive increase with the temperature. ${ }^{77-79} 458$

Figure $2 \mathrm{~b}$ plots the dependence, at different temperatures, of 459 the ionic conductivity from the solution molality. A bell 460 behavior is observed with a maximum value located between $c 461$ $=2$ and $5 \mathrm{~m}$, similar to the behavior observed for the LiTFSI- 462 $\mathrm{H}_{2} \mathrm{O}$ system. ${ }^{1,80}$ The conductivity $(\sigma)$ of electrolytes such as 463 the LiIM14- $\mathrm{H}_{2} \mathrm{O}$ solutions is governed by the following 464 equation

$$
\sigma=\sum_{i} n_{i} z_{i} \mu_{i}
$$

where $n_{i}$ represents the charge carrier number, $z_{i}$ is the ionic 466 charge, and $\mu$ is the mobility of the $i$ th ion species. At low 467 LiIM14 concentrations $(c<2 \mathrm{~m})$, the electrolyte conductivity 468 
469 is found to increase with the lithium salt molality due to the 470 increase of the charge carrier number. Also, the increase of the 471 LiIM14 molality leads to ion mobility decrease, but this effect 472 is fully counterbalanced by the increase of the charge carrier 473 number, overall enhancing the conductivity value. Conversely, 474 at higher concentrations $(c>5 \mathrm{~m})$, the increase of the lithium 475 salt molality leads to the formation of multiple ions and/or 476 neutral ionic couples, this progressive lowering of the overall 477 free charge carrier number and, therefore, the conduction value 478 of the water solution, thus leading to a maximum in 479 conductivity as the salt content increases. At low temperatures $480\left(T \leq 0{ }^{\circ} \mathrm{C}\right)$, the aqueous LiIM14 electrolytes show a maximum 481 conductivity around a salt molality of $5 \mathrm{~m}$ (at this condition, 482 some of the samples are still in the solid state), whereas above $48330{ }^{\circ} \mathrm{C}$ (i.e., when all solutions are in the molten state), such a 484 maximum value is seen shifting to $c=2 \mathrm{~m}$. It is to be noted 485 that all investigated LiIM14- $\mathrm{H}_{2} \mathrm{O}$ solutions exhibit ion 486 conduction values of interest for practical electrochemical 487 devices $\left(>10^{-3} \mathrm{~S} / \mathrm{cm}\right)$ at $-20{ }^{\circ} \mathrm{C}$, making these electrolyte 488 systems appealing for low-temperature applications.

489 Together with ion transport properties, electrochemical 490 stability is another important electrolyte property in view of its 491 application in practical devices. Figure 3 displays the anodic

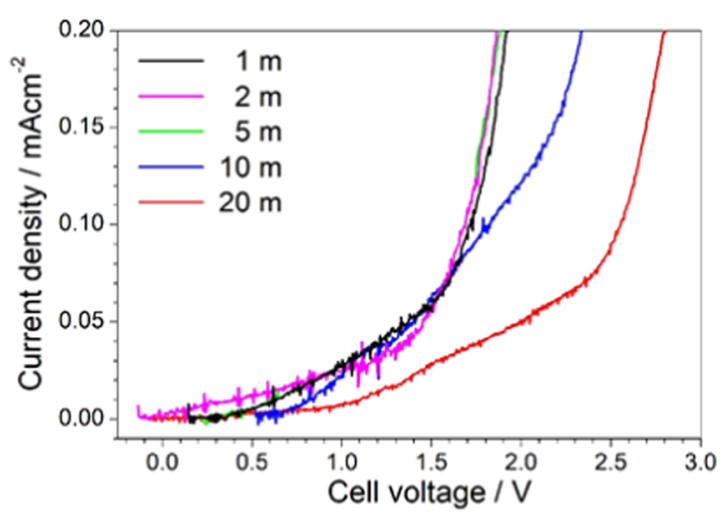

Figure 3. Anodic linear sweep voltammetry traces obtained for the LiIM14- $\mathrm{H}_{2} \mathrm{O}$ system at room temperature for different salt contents.

492 linear sweep voltammetry (LSV) traces obtained for the 493 investigated LiIM14- $\mathrm{H}_{2} \mathrm{O}$ systems. A sudden current increase, 494 observed in the voltage range from 1.5 to $2.5 \mathrm{~V}$, indicates 495 massive degradation (oxidation) of the electrolyte samples. 496 Similar electrochemical behavior (i.e., no practical improve497 ment in terms of anodic stability) is detected up to $c=5 \mathrm{~m}$, 498 whereas a progressive shift of the anodic limit voltage is 499 observed with the increase of the LiIM14 concentration above $500 c=5 \mathrm{~m}$. As known, the $\mathrm{Li}^{+}$cations, due to their high surface 501 charge density (ascribable to their small steric hindrance), can 502 strongly coordinate the polar water molecules. Thus, the 503 increase of the LiIM14 concentration leads to a progressive 504 decrease of the fraction of free (i.e., nonbounded to the lithium 505 salt) water molecules (vide infra). Up to $c=5 \mathrm{~m}$, the fraction 506 of free water within the electrolyte sample is remarkable and it 507 starts to degrade around $1.0 \mathrm{~V}$ according to the reaction (i.e., 508 redox potential equal to $-0.828 \mathrm{~V}$ vs $2 \mathrm{H}^{+} / \mathrm{H}_{2}$ )

$$
2 \mathrm{H}_{2} \mathrm{O}+4 \mathrm{e}^{-} \rightarrow 2 \mathrm{H}_{2}+2 \mathrm{OH}^{-}
$$

509 Above $c=5 \mathrm{~m}$, the free-water fraction is progressively 510 decreasing, and especially at very high salt molality values $(c=$ $51120 \mathrm{~m}$ ), all water molecules are practically involved in the lithium salt solvation (vide infra). Therefore, the voltammetry 512 results seem to provide support for the existence of a lithium 513 salt concentration threshold, which governs the electro- 514 chemical behavior of these highly concentrated aqueous 515 electrolyte systems. Below this threshold, the free-water 516 content is relevant and drives the anodic stability of the 517 solutions: no practical gain in terms of anodic limit voltage is 518 observed. Above this salt concentration, the free-solvent 519 fraction is negligible, i.e., almost the overall aqueous solvent 520 amount is involved in strong coordination of the $\mathrm{LiIM}_{14}$ salt 521 (especially of the $\mathrm{Li}^{+}$cations), and the anodic stability is found 522 to increase with increasing the lithium salt molality. 523 Spectroscopic and computational evidence in the next sections 524 will confirm this behavior. Therefore, very large LiIM14 525 concentrations, fully involving the whole aqueous solvent in 526 the solvation of ions, are able to shield the $\mathrm{H}_{2} \mathrm{O}$ molecules 527 from oxidation processes, thus enhancing the anodic stability 528 of the aqueous electrolytes. Similar behavior was previously 529 observed for concentrated aqueous solutions based on 530 LiTFSI. ${ }^{1}$ Therefore, even if the electrochemical stability is 531 not still sufficiently wide for applications in lithium battery 532 systems $^{81}$ operating at high voltages (i.e., above $4 \mathrm{~V}$ ), as also 533 reported in the literature, ${ }^{1}$ the very high molar concentration 534 of the LiIM14 salt is able to enhance the robustness of the 535 aqueous solution toward oxidation. Although other effects play 536 a role in the electrochemical stability of super-concentrated 537 WiS (see, e.g., ref 10), in any case, this feature makes the 538 present WiS system a promising class of materials for future 539 applications in electrochemical energy storage systems. ${ }^{2,3,8,11,12} 540$

The selected electrolyte system has been characterized in 541 terms of its density properties as a function of temperature 542 between 20 and $60{ }^{\circ} \mathrm{C}$. These data are reported in Figure S-2. 543 In the case of $c=20 \mathrm{~m}$, the sample at $20^{\circ} \mathrm{C}$ is in a supercooled 544 condition that could be reliably characterized, without 545 intervening crystallization, during the measurements. We next 546 probed morphological properties. Figure 4 shows the small- $547 \mathrm{f} 4$ angle X-ray scattering (SAXS) data sets collected for the series 548 of LiIM14-water in the concentration range between $c=1549$ and $20 \mathrm{~m}$ at ca. $20{ }^{\circ} \mathrm{C}$. The LiIM14-water SAXS patterns are 550

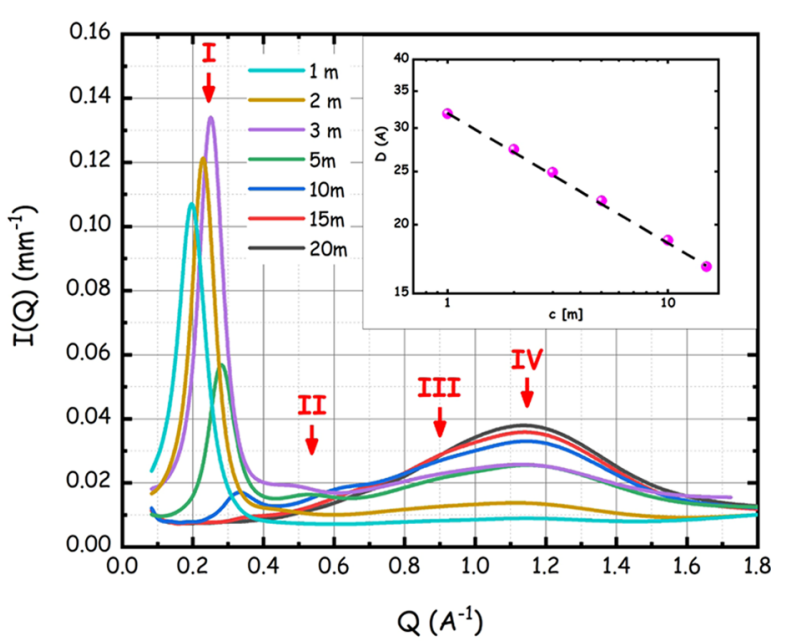

Figure 4. Small-angle X-ray scattering patterns for the LiIM14- $\mathrm{H}_{2} \mathrm{O}$ system at room conditions as a function of salt content. The roman numbers refer to the four different peaks observed in the patterns. In the inset, the $\log -\log$ salt concentration dependence for the characteristic size associated with peak I is reported. 
551 characterized by four peaks in the probed $Q$ range (a strong 552 peak (I) at $Q$ values $<0.4 \AA^{-1}$, one in the range of $0.4-0.7 \AA^{-1}$ 553 (II) and two peaks (III and IV) at $Q$ values above $0.7 \AA^{-1}$, as 554 outlined in the figure by the red arrows). It emerges clearly 555 that at a high/medium water content $(c \leq 10 \mathrm{~m})$, a very strong 556 scattering halo (peak I) develops at low $Q$ values $(Q<0.4$ $557 \AA^{-1}$ ). The intermediate peak (peak II) is appreciable, although 558 with weaker amplitude, across the whole concentration range. 559 Such a situation is different from the ones reported in the 560 recent past for the case of the LiTFSI-water system. Borodin 561 et al. observed a peak (likely corresponding to our present peak $562 \mathrm{I}$ ) in LiTFSI WiS (with $c=21 \mathrm{~m}$, with $\mathrm{D}_{2} \mathrm{O}$ ), using small-angle 563 neutron scattering data. ${ }^{13}$ Recently, a report from Zhang et al. 564 showed high-energy X-ray scattering data from a series of 565 LiTFSI-water systems in the concentration range between 1 566 and $20 \mathrm{~m}^{28}$ Their data allow detecting the presence of two 567 peaks in the range between 0.2 and $2 \AA^{-1}$. These data are 568 similar to the ones reported by Liu et al. for the same system. ${ }^{55}$ 569 The neutron weighted simulated patterns reported by Borodin 570 et al. for LiTFSI WiS, with the concentration ranging from 5 571 up to $21 \mathrm{~m}$, indicate a progressive growth in the amplitude of 572 the low $Q$ feature but their simulations do not seem to indicate 573 an appreciable change in the peak position, between $c=5$ and $57421 \mathrm{~m}$. ${ }^{13}$ The data presented by Zhang et al. do not allow 575 detecting this behavior, as they are vertically shifted; they 576 observe, however, a distinct shift in the peak position. ${ }^{28}$ Very 577 recently, Tan et al. reported neutron and X-ray scattering data 578 from the LiTFSI-water system at $c=0.3$ and $21 \mathrm{~m}^{22}$ Their 579 study highlighted the presence of peak I (centered at $0.4 \AA^{-1}$ ) 580 in concentrated solution using both $\mathrm{X}$-ray and neutron 581 scattering, but they claim that peak I is not present in dilute 582 solutions. Horwitz et al. monitored peak I evolution between $c$ $583=4$ and $21 \mathrm{~m}$ for LiTFSI-water by neutron scattering using $584 \mathrm{D}_{2} \mathrm{O} .{ }^{48}$ Our present results on LiIM14-water electrolytes 585 indicate the progressive development of the distinct low $Q \mathrm{X}$ 586 ray scattering peak I upon increasing the water content, whose 587 position clearly shifts with the concentration. In Figure S-3, we 588 show the data of Figure 4 in $\log -\log$ scale and highlight the 589 concentration dependence of peaks positions. It emerges that 590 peaks III and IV positions show only a minor concentration 591 dependence. On the other hand, both peaks I and II 592 appreciably shift toward higher $Q$ values upon increasing the 593 salt content. This behavior is similar to what Zhang et al. 594 reported for their peaks B and A, respectively, in their paper. ${ }^{28}$ 595 The behavior is also similar to the one highlighted by Horwitz 596 et al. in their recent paper. ${ }^{48}$

597 Peak positions for peak I, $Q_{p}$, have been determined as a 598 function of salt concentration by fitting the experimental data 599 with a Gaussian function and the corresponding real space 600 sizes estimated as $D=2 \pi / Q_{p}$ are reported in the inset of Figure 6014 . The linear trend of $\log D$ vs $\log c$ in the concentration range $602(1 \leq c([\mathrm{~m}]) \leq 15)$ probed by the present study can be 603 noticed. The concentration dependence of peak I amplitude is 604 noteworthy; while our data show that the peak occurs 605 ubiquitously in the probed concentration window, its 606 amplitude shows a maximum at ca. $c=3 \mathrm{~m}$. Accordingly, at 607 odds with the observation done by Tan et al., ${ }^{22}$ the structural 608 heterogeneities leading to the appearance of peak I are present 609 over the whole probed concentration range. In this context, we 610 also mention the recent report from Liu et al., where a large set 611 of concentrations of LiTFSI-water mixtures has been studied 612 by small-angle X-ray scattering (SAXS), confirming our present 613 findings that peak $\mathrm{I}$ is stronger at more dilute conditions and, by increasing the salt content, its amplitude tends to decrease 614 (and even vanish) and its position shifts to higher $Q$ values. ${ }^{55} 615$

Overall, then, we can state that, apart from the peak 616 amplitude, we do not observe a drastic differentiation between 617 salt-in-water and water-in-salt regimes concerning the low $Q 618$ peak in this class of material. Such an observation is important 619 to properly address the attention in the exploration of lithium 620 diffusivity in WiS. The present results indicate the existence of 621 structural heterogeneities of the order of several nanometers, as 622 revealed by X-ray scattering, whose size depends on the 623 electrolyte composition. Such heterogeneities are present 624 across the explored concentration window in the present 625 electrolytes. This scenario will be confirmed by molecular 626 dynamics simulations later on. At the present stage, we can, 627 however, propose the existence of a sponge-like, bicontinuous 628 morphology that characterizes the mutual distribution of self- 629 excluding domains of apolar, fluorinated anions and water in 630 these electrolyte systems.

631

The series of LiIM14-water samples have also been 632 characterized by synchrotron high-energy X-ray diffraction, 633 aiming at accessing a larger $Q$ range than the one accessible via 634 the SAXS technique. These data are of course compatible with 635 the SAXS ones in their common $Q$ range, but they also provide 636 information on shorter-range structural correlations occurring 637 in the liquid samples by accessing $Q$ values as high as $20 \AA^{-1} .638$ Typically, these data sets are used to provide experimental 639 validation of the structural properties as extracted via 640 molecular dynamics simulation, which can be judged by the 641 quality of the agreement between experimentally and computa- 642 tionally derived static structure factors, $S(Q)$.

Figure 5 reports such a comparison between the measured $644 \mathrm{fs}$ $S(Q)$ (over the range $0.1 \leq Q\left(\AA^{-1}\right) \leq 8$ ) and the 645

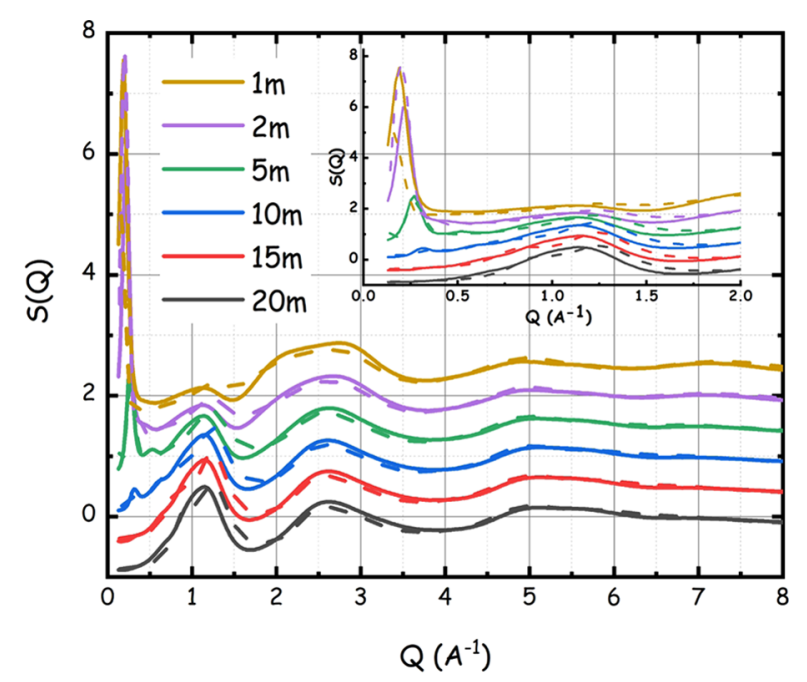

Figure 5. Experimental (continuous lines) and MD-derived (dashed lines) wide-angle X-ray scattering patterns from the LiIM14- $\mathrm{H}_{2} \mathrm{O}$ system for different salt contents at ambient conditions. In the inset, the low $Q$ portion of the spectra is highlighted.

corresponding patterns as obtained from the MD simulations. 646 The latter nicely account for all of the relevant experimental 647 features and, especially, for the emerging of the strong peak at 648 low $Q$ values, upon diluting the mixtures. We stress that to 649 satisfactorily reproduce the low $Q$ scattering features, a large 650 simulation box is required. Here, the use of box sizes of the 651 order of $80-100 \AA$ turned out to be fundamental for the 652 
(a)

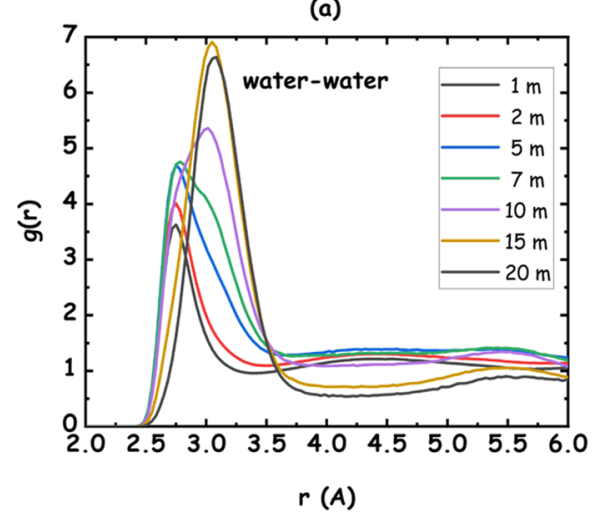

(c)

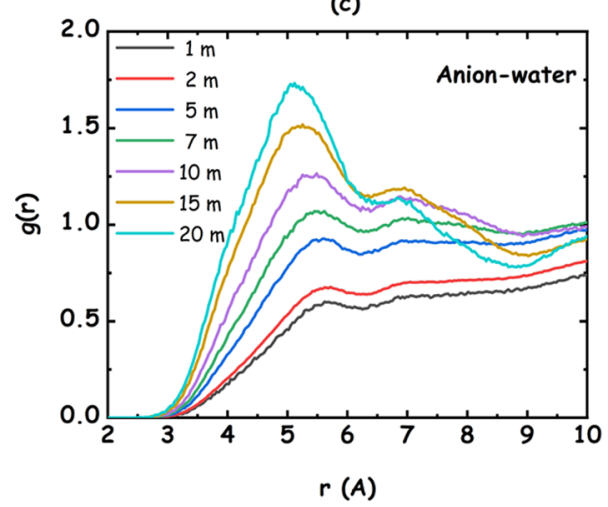

(e)

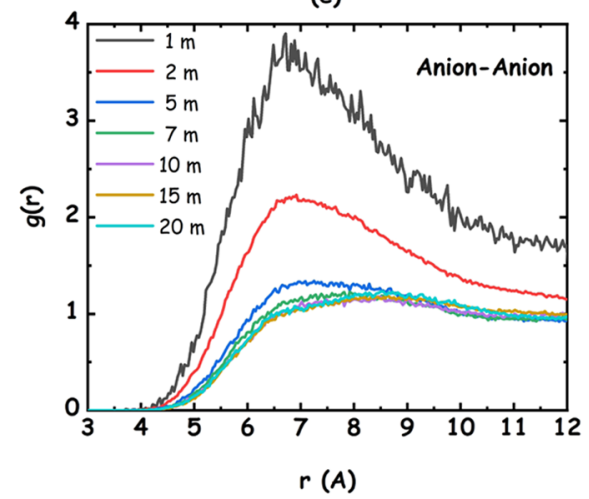

(b)

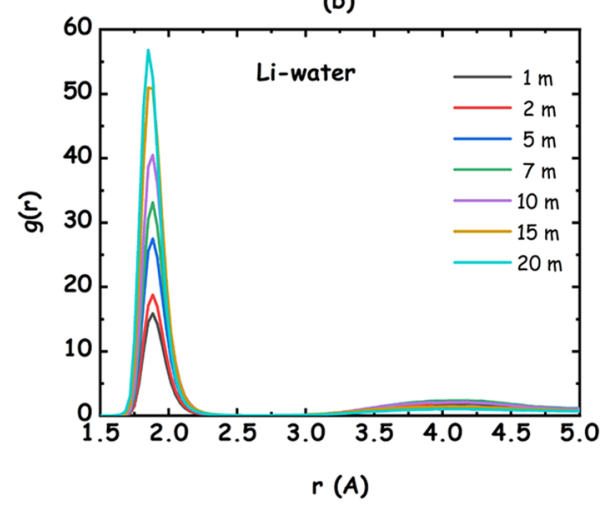

(d)

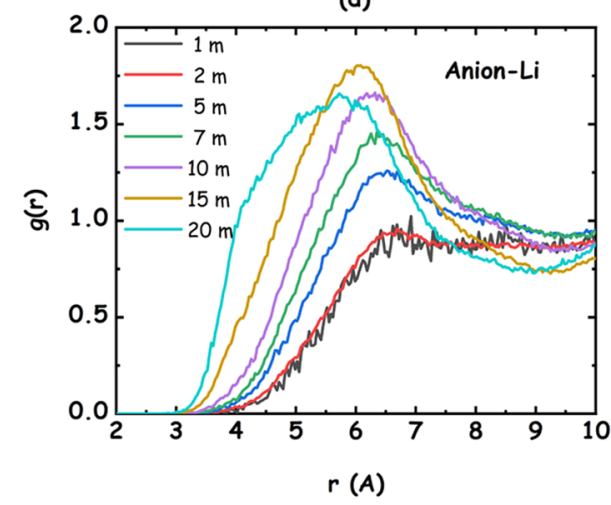

(f)

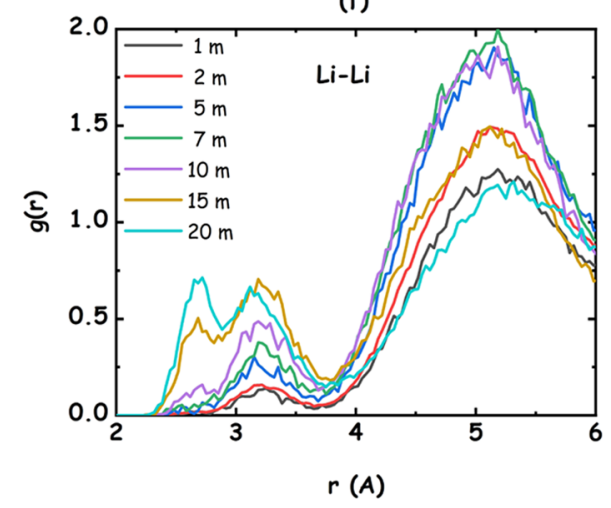

Figure 6. MD-derived center of mass pair distribution functions for the different species in the LiIM14- $\mathrm{H}_{2} \mathrm{O}$ systems, for different salt contents: (a) water-water; (b) lithium-water; (c) anion-water; (d) lithium-anion; (e) anion-anion, and (f) lithium-lithium correlations are shown.

653 purpose. More conventional sizes (e.g., 30-50 ̊̊) would either 654 miss to reproduce or wrongly estimate the position and 655 amplitude of such features.

656 For the sake of completeness, in Figure S-4, we report our 657 molecular dynamics computed $S(Q)$ as would be obtained 658 from a neutron scattering experiment using either $\mathrm{H}_{2} \mathrm{O}$ or ${ }_{659} \mathrm{D}_{2} \mathrm{O}$. Therein, one can appreciate the ubiquitous presence of 660 peak I over the whole probed concentration window, thus 661 supporting the above discussion.

662 Together with the comparison between experimental and 663 computed X-ray scattering patterns, we further validated the 664 presently reported $\mathrm{MD}$ simulations with the experimental 665 values of density at $25{ }^{\circ} \mathrm{C}$. The agreement is very good and is 666 reported in Figure S-5. This robust experimental validation of 667 the simulations makes us confidant in their exploitation for 668 extracting accurate structural information at the atomistic level.
As preliminary information, we interrogated the MD 669 simulations to extract the pair distribution functions (PDF) 670 related to the three species centers of mass (CoM) mutual 671 correlations for the different investigated WiS. These data are 672 plotted in Figure 6a-f, where the concentration dependence of $673 \mathrm{fo}$ the self and cross-correlations are reported for the three 674 species: namely, water, lithium, and the [IM14] anion. Other 675 relevant PDFs related to interatomic correlations are presented 676 in Figure 7 (corresponding figures containing the running $677 \mathrm{f7}$ coordination numbers are reported in Figures S-6 and S-7). 678

In general, the observed trends tend to be similar to recent 679 results from two different groups, which focused on structural 680 properties of LiTFSI-water system.

681

Water-Water Correlations. Considering the case of 682 water-water correlations (Figure 6a), while dilute mixtures 683 are characterized by a simple peak centered at $2.75 \AA$, 684 reflecting bulk water's tetrahedral organization, on the other 685 
(a)

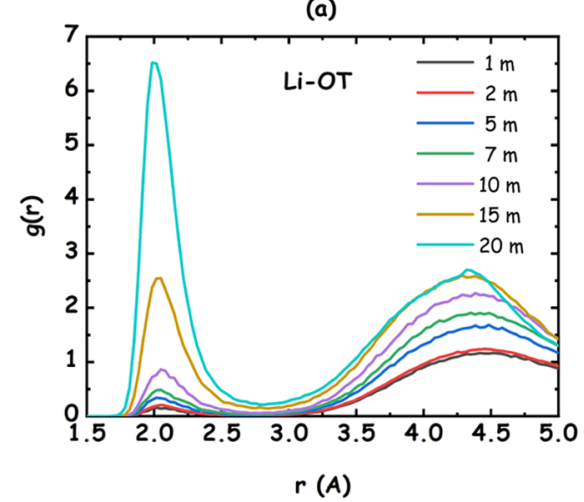

(c)

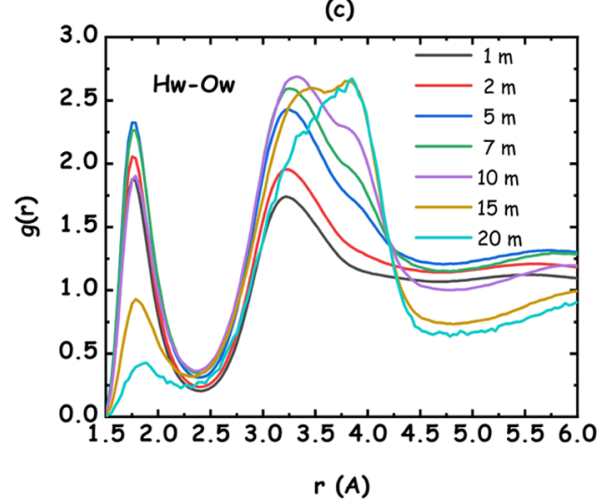

(e)

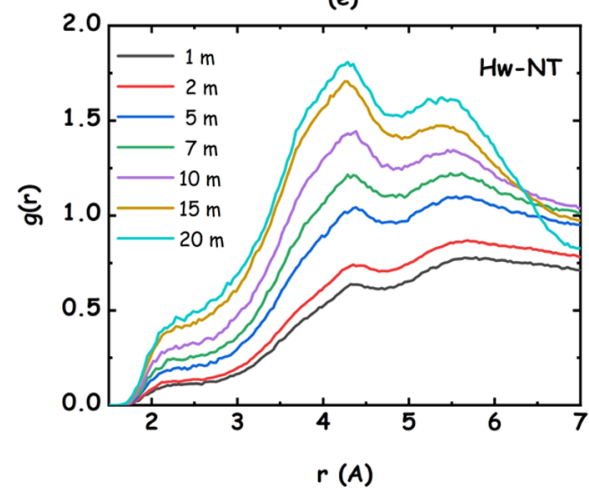

(g)

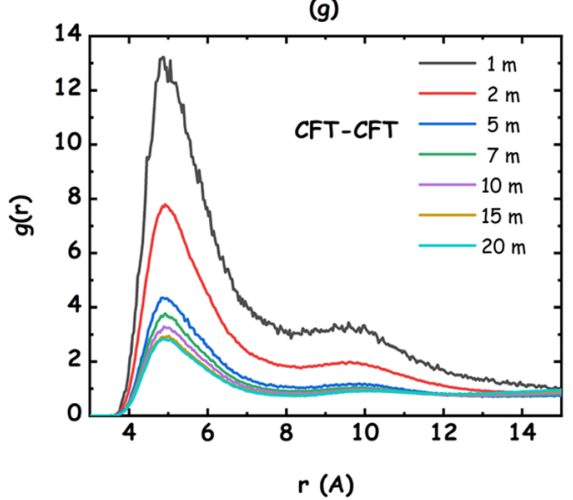

(b)

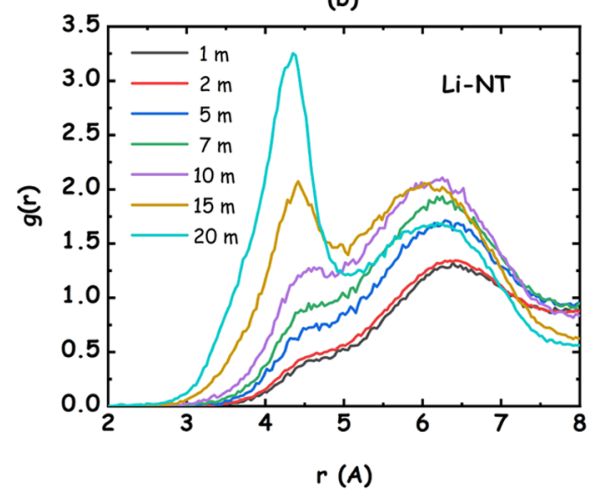

(d)

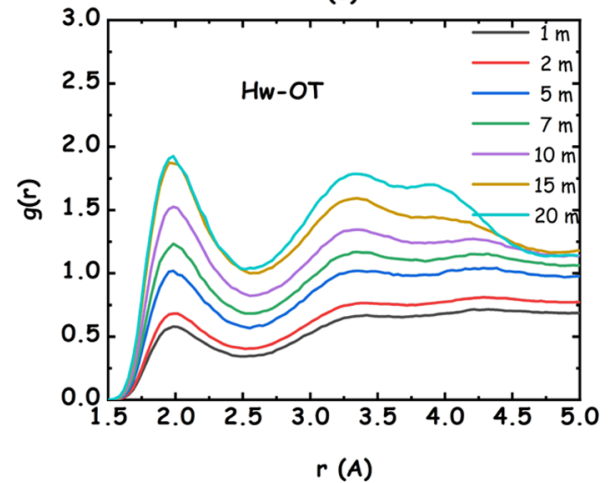

(f)

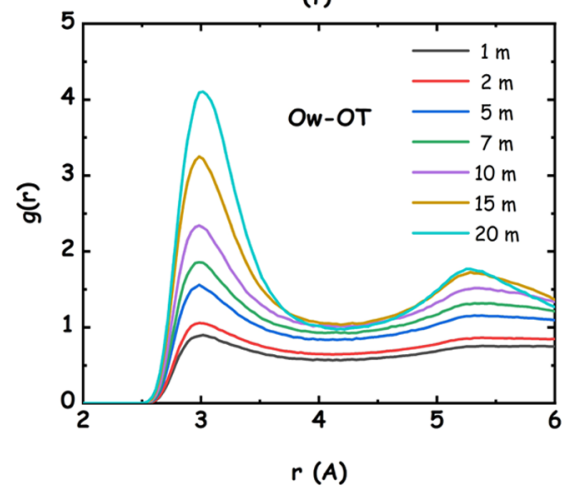

Figure 7. Selected, MD-derived pair distribution functions for the different species in the LiIM14- $\mathrm{H}_{2} \mathrm{O}$ systems, for different salt contents: (a) Li$\mathrm{OT}$; (b) Li-NT; (c) Ow-Hw; (d) OT-Hw; (e) NT-Hw; (f) OT-Ow, and (g) CFT-CFT correlations are shown. Ow and Hw refer to water's oxygen and hydrogen atoms; OT, NT, and CFT refer to anion's oxygen, nitrogen, and terminal butyl carbon atomic species (see Figure 1).

686 hand, upon increasing the salt concentration, a progressively 687 bimodal distribution (that is clearly visible at the two 688 concentrations $c=7$ and $10 \mathrm{~m}$ ) will eventually evolve into a single peak centered at $3.1 \AA$, reflecting a change in water- 689 water correlations. Following the rationalization for this 690 behavior provided by Zhang et al., ${ }^{28}$ we observe that in pure 691 
692 water and in dilute LiIM14 mixtures, the conventional 693 tetrahedral organization of water molecules surrounding a 694 reference water molecule is reflected by the peaks at 2.75 and $6954.5 \AA$. On the other hand, the progressively increasing lithium 696 content will lead to a decrease of the bulk-water population 697 and the development of lithium-mediated water-water 698 correlations with a characteristic water-water distance of the 699 order of $3.2 \AA^{28}$ It is important to note that lithium-mediated 700 neighbor waters are not directly interacting through the 701 hydrogen-bonding interaction between themselves. Accord702 ingly, the shift and splitting of the water-water PDF peak 703 reflects a progressive change in the nature of water environ704 ments in the solutions. In Figure S-8, we show the distribution 705 numbers of water oxygens, Ow, coordinating a reference Ow, 706 as a function of salt content. Figure S-8a shows the distribution 707 of Ow coordination numbers obtained inside a shell of $3.3 \AA$ 708 (that is, the typical $\mathrm{O} \cdots \mathrm{O}$ distance between hydrogen-bonded 709 Ow's in bulk water). One can notice that upon increasing the 710 salt content, the local water environment remains appreciably 711 uninfluenced up to $c=2 \mathrm{~m}$; above this value, one notices a 712 progressive shift toward a smaller number of coordinating 713 waters. Figure $\mathrm{S}-8 \mathrm{~b}$ highlights that the average $\mathrm{Ow}$ 714 coordination number around a reference $\mathrm{Ow}$ is found to 715 progressively decrease down to ca. 2 when increasing the salt 716 content. Moreover, one finds a dramatic increase of the 717 number of reference Ow's with no surrounding hydrogen718 bonded water molecules, with $50 \%$ water molecules not bound 719 to any other one via $\mathrm{HB}$, already at $c=10 \mathrm{~m}$ (Figure S-8c). 720 Such a situation is reflected by the concentration trend 721 observed for the orientational tetrahedral order (OTO) 722 parameter $^{82}$ that is reported in Figure S-9. Therein one can 723 observe that dilute solutions are characterized by a water 724 network surrounding a reference water molecule resembling 725 the tetrahedral order observed in neat water. However, when 726 the salt content increases, the OTO parameter strongly 727 deviates from the neat water behavior.

728 Water-Lithium Correlations. Lithium-water PDFs are 729 characterized by a strong peak centered at ca. $1.9 \AA$ (Figure $7306 \mathrm{~b})$. Over the probed salt concentration range, the lithium 731 cation tends to maintain water coordination (Figure S-6b) and 732 the resulting water molecules surrounding lithium will organize 733 with a mutual reciprocal distance of ca. $3.2 \AA$ (see above). Such 734 an interaction will strongly affect water organization that, 735 accordingly, shows drastic evolution, as mentioned above. In 736 Figure S-10, we show the coordination number distributions 737 and the average coordination numbers of water hydrogens, $738 \mathrm{Hw}$, and lithium cations surrounding a reference water oxygen, 739 Ow. Clearly, there is a competition between the two species in 740 solvating Ow. The number of $\mathrm{Hw}$ surrounding Ow decreases 741 from 1.7 in neat water down to ca. 0.1 at $c=20 \mathrm{~m}$; conversely, 742 lithium progressively increases its solvation number up to 1 ion 743 at $c=20 \mathrm{~m}$, reflecting the change in coordination of water and 744 the strong ability of lithium ions to coordinate water.

745 Water-Anion Correlations. Water also interacts with the 746 sulfamide portion of the anion via hydrogen-bonding 747 interactions. Water hydrogen-anion oxygen (Hw-OT) and 748 water hydrogen-water oxygen $(\mathrm{Hw}-\mathrm{Ow})$ correlations are both 749 influenced by the change in the salt content. Figure $7 c, d$ shows 750 the evolution of corresponding PDFs. Both PDFs are 751 characterized by a distinct peak at 1.8 and $2.0 \AA$, respectively. 752 The H-bonds involving either Ow or OT as acceptor ones are 753 characterized by a short $\mathrm{Hw} \cdots \mathrm{Ox}$ distance and a rather linear 754 geometry $\left(\mathrm{Ow}-\mathrm{Hw} \cdots \mathrm{Ox}>150^{\circ}\right.$ (for $\mathrm{Ox}=\mathrm{Ow}$ and $\mathrm{OT}$ ) (data not shown)). By integrating the above-mentioned PDFs, one 755 notices that upon increasing the salt content, the number of 756 Ow coordinating $\mathrm{Hw}$ decreases from 1 to less than 0.1 , while 757 an increase of the number of OT coordinating each $\mathrm{Hw}$ is 758 found up to 0.6 at $c=20 \mathrm{~m}$ concentration (see Figure S-11). 759 Overall, the oxygen coordination (whatever its origin, either 760 water or anion) toward water hydrogen decreases from ca. 1761 down to 0.7 , reflecting a substantial change in water 762 coordination organization. As reflected by Figure 7e, the 763 anion nitrogen is strongly hindered from the interaction with 764 water by the bulky $\mathrm{SO}_{2}$ groups; accordingly, the anion interacts 765 with water only through its OT atoms.

766

Overall, upon increasing the salt content, the water solvation 767 environment dramatically changes. Figure 8 shows the $768 \mathrm{f} 8$

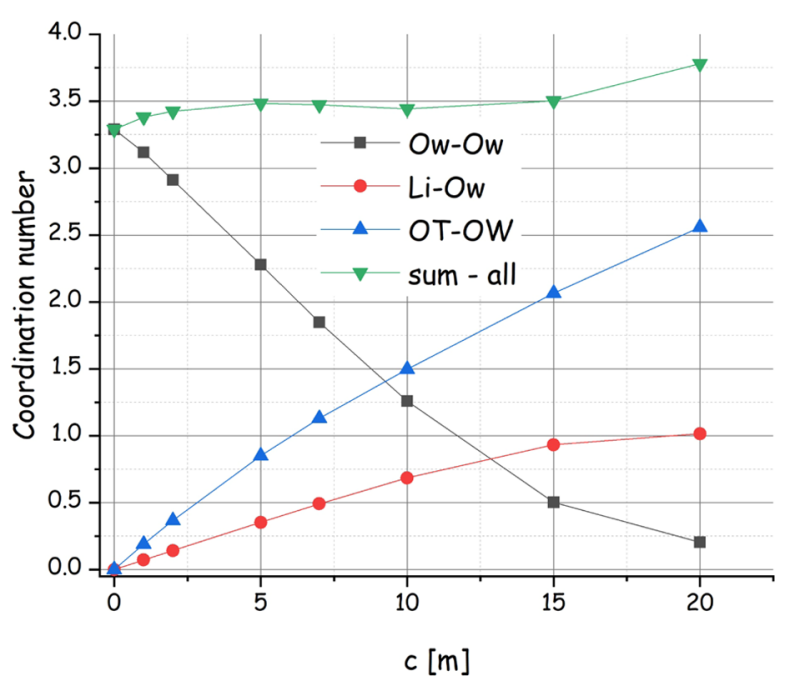

Figure 8. Salt content dependence of the coordination number of water's oxygen, lithium, and anion oxygen around a reference water oxygen, as obtained by the MD simulations of the LilM14- $\mathrm{H}_{2} \mathrm{O}$ system.

composition of the surrounding environment around a 769 reference water oxygen, decomposing it into water molecules 770 (bound to the reference one either via HB-donor or HB- 771 acceptor interactions), lithium cations, and anion's oxygen 772 atoms. Upon increasing the salt content, the number of water 773 molecules decreases down to a minimum value of 0.25 , and, 774 correspondingly, one observes an increase of lithium (up to 775 one ion) and anion oxygen (up to 2.5) solvation of the 776 reference water molecule. The sum of the solvating moieties 777 remains pretty much constant to ca. 3.5. In this scenario, the 778 number of coordinating water molecules drastically decreases 779 not only as a consequence of the smaller water content but also 780 due to their replacement by either lithium or anion oxygen. 781 Accordingly, the ability of the $\mathrm{HB}$ acceptor toward water is 782 essentially lost (a negligible amount of $\mathrm{Hw}$ approaching the 783 reference $\mathrm{Ow}$ ), and the ability of the $\mathrm{HB}$ donor drastically 784 decreases and involves anion OT rather than Ow.

785

Ionic Species Correlations. Despite the strong interaction 786 between lithium and water, the sulfamide moiety of the anion 787 is a competitor with $\mathrm{Ow}$ toward lithium coordination. Figure 788 $6 \mathrm{~d}$ shows the evolution of Li-anion correlations upon 789 increasing the salt content: dilute solutions are characterized 790 by a PDF with an amplitude below one, over more than $10 \AA, 791$ indicating that the ions are on average fully solvated by water 792 and a very limited amount of contact ion pairs (CIP) exists. 793 


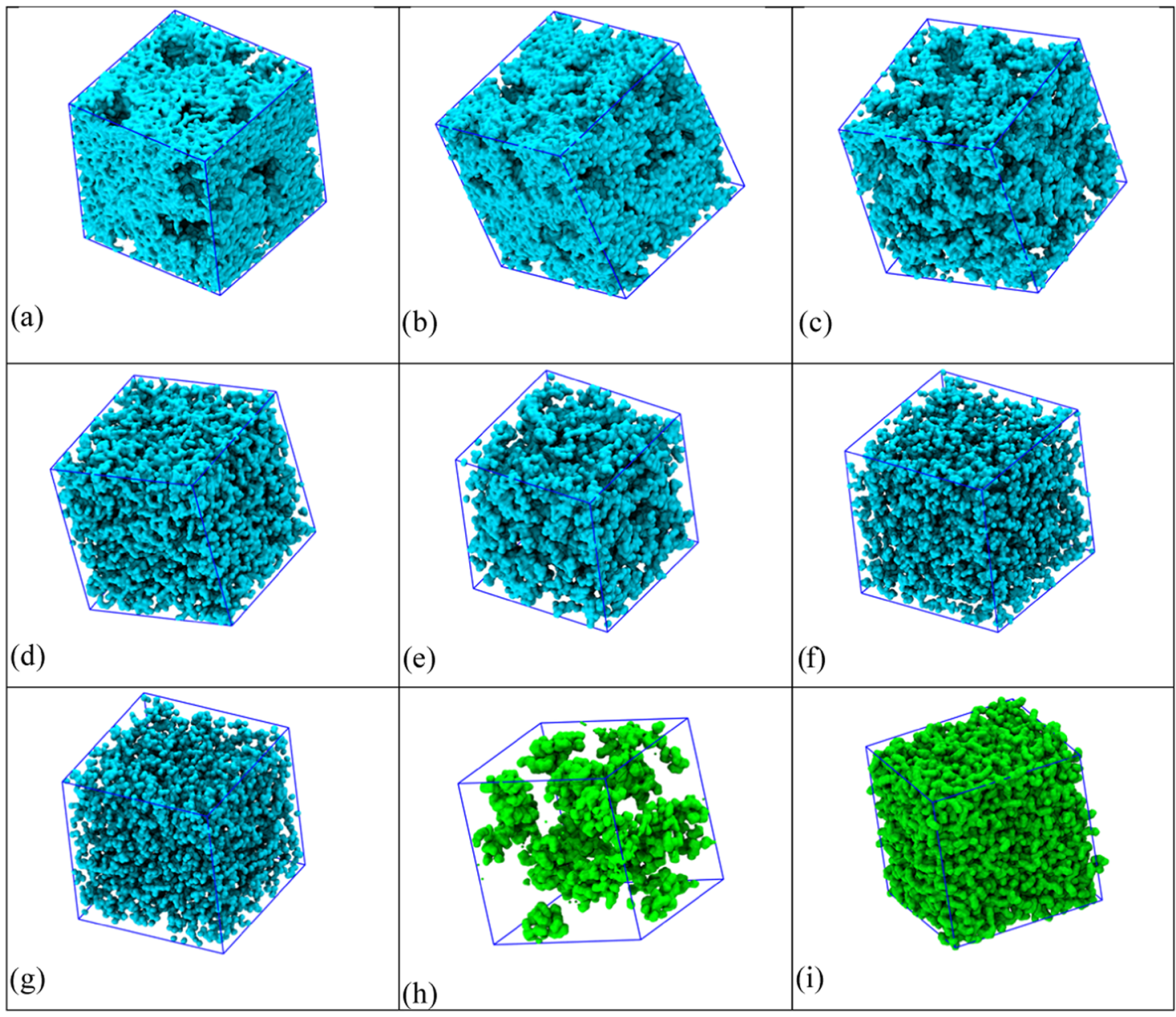

Figure 9. Simulated snapshots of the LiIM14- $\mathrm{H}_{2} \mathrm{O}$ system, where only water is shown $((\mathrm{a}-\mathrm{g})$ for $c=1,2,5,7,10,15$, and $20 \mathrm{~m}$, respectively) and where only the anions are shown $((\mathrm{h}, \mathrm{i})$ for $c=1$ and $20 \mathrm{~m}$, respectively). Box sizes vary in the range of $85-105 \AA$.

794 Already at concentrations as high as $c=5 \mathrm{~m}$, however, a peak 795 manifests at ca. $6.5 \AA$ and its position and amplitude evolve 796 with the increasing salt content. In particular, the Li-anion 797 mutual distance progressively decreases and the number of 798 neighbors increases (Figure S-6d), indicating the development 799 of direct Li-anion correlations: these manifest themselves 800 through the $\mathrm{Li}-\mathrm{OT}$ interactions and are in competition with $801 \mathrm{Li}-\mathrm{Ow}$ correlations. In Figure $\mathrm{S}-12$, we show both the 802 coordination distribution numbers and the average number of 803 either Ow or OT coordinating a reference lithium ion as a 804 function of salt content. It is noticeable that the average 805 number of oxygen atoms (whatever their origin, either water or 806 anion) surrounding the reference lithium ion remains 807 appreciably constant and equal to four. This occurs with a 808 progressive decrease of Ow and an increase of OT belonging 809 to the first lithium solvation shell upon increasing the salt 810 content. It has been noticed previously that even at the highest 811 salt content $(c=21 \mathrm{~m})$, a non-negligible fraction of $\mathrm{Li}_{(}\left(\mathrm{H}_{2} \mathrm{O}\right)_{4}{ }^{+}$ 812 clusters exists for the case of LiTFSI electrolytes. ${ }^{13,30}$ Here, we 813 observe that also in the case of LiIM14 WiS at $c=20 \mathrm{~m}$, ca. $81420 \%$ of lithium is coordinated by four water molecules with an 815 average coordination number of ca. 2.5 water molecules. Such 816 entities are considered to be fundamental in determining the 817 peculiar conductivity performances of such a class of WiS, 818 allowing lithium ions to diffuse uncoupled from the anions. 819 Consistently, at the same extreme concentration, a fraction of
$25 \%$ lithium ions appears not to be coordinated by any anion 820 oxygen. There is also a negligible population corresponding to 821 lithium solvation by more than two OT's. This is at odds with 822 the behavior observed in the case of the TFSI anion: ${ }^{13}$ in 823 particular, we do not find support in the case of the present 824 anion for the rather extreme behavior of lithium that either 825 prefers to be solvated by four water molecules or by four anion 826 oxygens. $^{13}$ Our results indicate an extreme preference of 827 lithium for water coordination rather than anion; presumably, 828 this is due to the different anion sizes that sterically hinder 829 specific interactions in the present case of the IM14 anion. $\quad 830$

Lithium-anion nitrogen (NT) correlations also develop but 831 rather as a consequence of the interaction between lithium and 832 the sulfamide moiety than as a direct interaction. To clarify the 833 matter concerning the formation of contact ion pairs (CIP) in 834 alternative to solvent-separated ion pair (SSIP) as a function of 835 salt content, we monitored the probability of the lithium ion to 836 be coordinated by a given number of either anion nitrogen (by 837 a distance of $5 \AA$ ) or anion oxygen (by a distance of $2.7 \AA$ ). 838 These distributions of coordination numbers are plotted in 839 Figure S-13, together with the corresponding percentages of 840 no-coordination occurrence for the case of nitrogen and 841 oxygen that represent two related evaluations of the fraction of 842 SSIP occurrence. The observed trend recalls the one observed 843 by Suo et al. ${ }^{13}$ for the case of LiTFSI WiS: as it was stressed 844 therein, the reported quantities represent a lower bound 845 


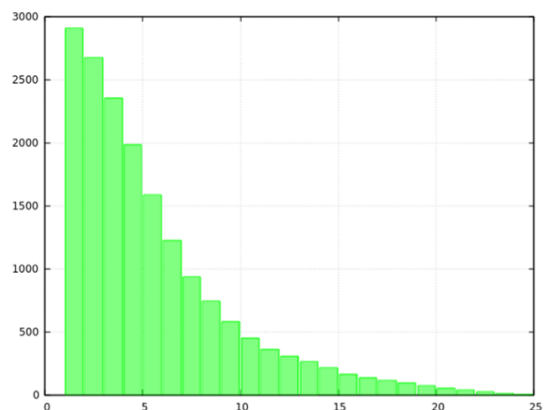

(a)

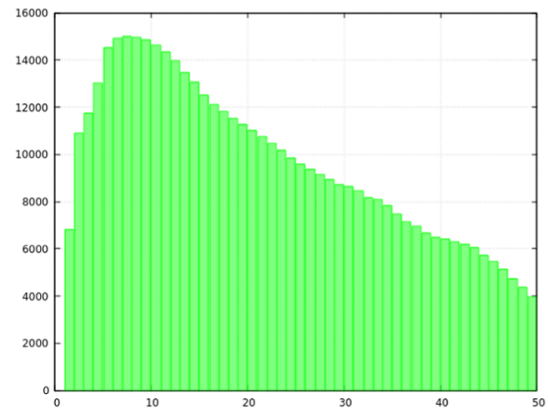

(d)

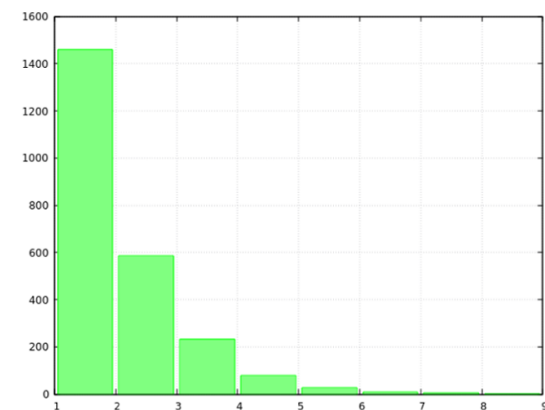

(b)

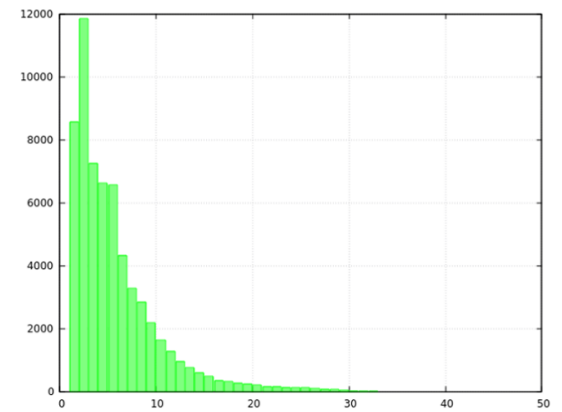

(e)

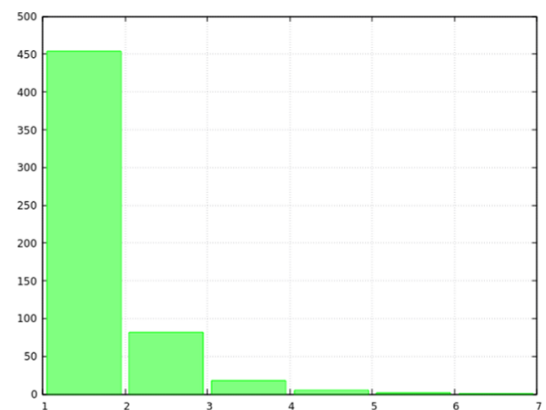

(c)

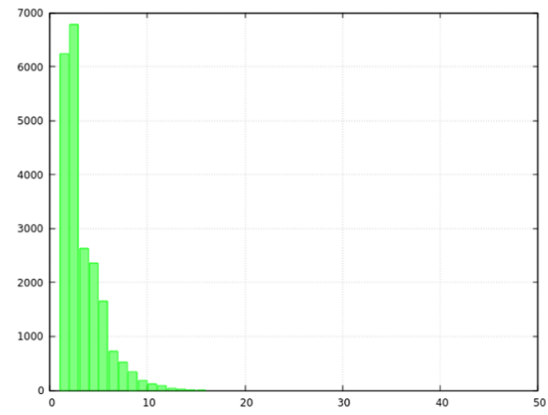

(f)

Figure 10. Distribution of chain element numbers for: (top) HB interacting water molecules in LiIM14 WiS, with $c=(a) 10 \mathrm{~m},(\mathrm{~b}) 15 \mathrm{~m}$, and (c) $20 \mathrm{~m}$ and (bottom) HB interacting water molecules and/or Li-Ow interactions in LiIM14 WiS, with $c=(\mathrm{d}) 10 \mathrm{~m},(\mathrm{e}) 15 \mathrm{~m}$, and (f) $20 \mathrm{~m}$.

846 estimate of the SSIP. It emerges that the fraction of CIPs 847 (complementary to the SSIP fraction) is very small at low 848 water content, and it increases with the salt amount. Overall, 849 the present results for LiIM14 electrolytes indicate a somehow 850 higher SSIP fraction at the highest concentration than 851 observed for the case of TFSI. ${ }^{13}$ In agreement with the 852 previous discussion, lithium is mainly solvated by water at 853 dilute conditions and a dominant population of SSIP 854 characterizes the ionic species organization. With increasing 855 the salt content, the water solvation around lithium 856 progressively diminishes and a corresponding fraction of 857 CIPs can be appreciated.

858 Lithium-lithium correlations (Figure 6f) are rather weak, 859 seemingly due to electrostatic reasons, and only at high salt 860 content can one detect the development of a close approach 861 that, however, correspond to very small coordination numbers. 862 Anion-anion correlations (Figure 6e) are also weak. The 863 occurrence of terminal perfluorobutyl $-\mathrm{CF}_{3}$ group clustering 864 (Figure $7 \mathrm{~g}$ ) that leads to a constant value of ca. 6 neighbor 865 groups surrounding the reference one, in its first solvation 866 shell, across the whole salt content range is noticeable (Figure $867 \mathrm{~S}-7 \mathrm{~g}$ ). Such a situation implies the occurrence of anion 868 clustering due to hydrophobic correlations between the 869 fluorous tails, analogously to the case of ionic liquids bearing 870 long fluorinated chains. ${ }^{51,83-85}$

871 Mesoscopic Organization. Figure 9a-i reports a pictorial 872 view of the simulated boxes after completion of the 873 computations. Therein (Figure $9 \mathrm{a}-\mathrm{g}$ ), only water molecules 874 are shown and empty spaces are filled by anions and lithium 875 ions. The two extreme cases $(c=1$ and $20 \mathrm{~m})$ are also shown 876 for the complementary case, i.e., showing only the anions and 877 no water/lithium species, for ease of comparison (Figure 9h,i). 878 Inspection of these figures can provide a useful rationalization 879 for several observations done across the manuscript so far. One 880 can appreciate the origin of the peculiarly evident low $Q$ features in the X-ray/neutron scattering patterns. In fact, such 881 features are much more intense than reported for the case of 882 the LiTFSI-water system, likely due to the larger size of the 883 fluorinated apolar portion of the anion. At dilute concen- 884 trations (between $c=1$ and $5 \mathrm{~m}$ ), water constitutes a 885 homogeneous matrix, with a percolating hydrogen-bonding 886 network connecting water molecules. Inside this homogeneous 887 environment, the hydrophobic anions segregate into essentially 888 globular domains, as can be appreciated by comparison of the 889 snapshots reported for the case $c=1 \mathrm{~m}$ when either only water 890 or only IM14 are plotted (Figure 9a,h, respectively). Ionic 891 species (both Li and IM14) are fully solvated by water, and the 892 fluorinated tails are mutually interacting through dispersive 893 interactions. The increasing salt content leads to the 894 progressive merging of the anion domains, with a high degree 895 of interpenetration of the two micro-segregated phases (Figure 896 $9 \mathrm{~d}, \mathrm{e})$. At intermediate concentrations, indications of channel- 897 like morphologies built up by water molecules appear, 898 consistently with past observations in the literature. ${ }^{21} 899$ However, the situation changes further at the highest salt 900 content mixtures, where a finely interpenetrated morphology is 901 observed without evidence of the claimed water channels. ${ }^{21}$ At 902 a high salt content, the anions form a percolating network 903 (Figure 9i) held up by anion- $\mathrm{Li}$ and anion-water interactions. 904 As water and lithium are fluorophobic, an efficient anion 905 solvation cannot be achieved and the anions interact with these 906 species mostly through their imide moiety, while the 907 fluorinated moieties remain segregated. Water then efficiently 908 interacts with both lithium and the polar part of the anions, 909 thus creating a finely dispersed aqueous mesh adhering to the 910 anion matrix, with very limited contact with other water 911 molecules. Further indication on the nature of such 912 mesoscopic organization of water molecules in these systems 913 has been obtained by monitoring the spatial extent of chains 914 built up either by hydrogen-bonded water molecules or by a 915 
916 joint interaction between $\mathrm{H}$-bonded water molecules and those 917 connected via a lithium cation. Figure $10 a-c, d-f$ shows such 918 distributions of component numbers for either water-water 919 only or water-water + water-lithium correlated chains, 920 respectively, for the case of the most concentrated solutions. 921 One detects that dilute solutions (not shown) are 922 characterized by long water-only chains percolating across 923 the simulation box. However, when above $c=10 \mathrm{~m}$, the 924 number of HB-connected water molecules drops significantly 925 and the $c=20 \mathrm{~m}$ system is characterized by just a few $(1-2)$ 926 water molecules that are $\mathrm{HB}$ connected to a reference one: no 927 longer chains are appreciable (see Figure 10c). On the other 928 hand, lithium-mediated connections between water molecules 929 show a substantially larger spatial extent. These structures can 930 probably be considered involving those lithium ions that can 931 efficiently migrate and transfer charge across the system. ${ }^{13,21}$ 932 The case of $c=10 \mathrm{~m}$ shows a very broad distribution for the 933 length of such mixed water-lithium chains; however, the plots 934 for more concentrated solutions prompt that no clear 935 indication of percolating channels that might be responsible 936 for lithium flow in salt-rich mixtures appears. These chains are 937 constituted by max. 20 or even $<10$ members (for $c=15$ and $93820 \mathrm{~m}$, respectively), which is too small a number to guarantee 939 percolation effects. Overall, the comparison between Figure 4 940 and Figure $9 \mathrm{a}-\mathrm{i}$ and similar plots from related papers indicates 941 that dilute solutions are characterized by a strong low $Q$ 942 scattering peak that is due to the formation of globular entities 943 formed by segregated anions into the water matrix. They are 944 very large: typically $1-3 \mathrm{~nm}$. Upon increasing the salt content, 945 local electroneutrality and increasing fluorous tail content lead 946 to a progressive merging of these globules into a three947 dimensional matrix that eventually, at the highest concen948 trations, will percolate across the simulation box. Our present 949 results suggest that the low $Q$ peak is the fingerprint of 950 alternating anion and water domains, as the $S(Q)$ decom951 position into different contributions leads to water-water and 952 anion-anion peaks out of phase with water-anion anti-peaks 953 (data not shown). ${ }^{22,28}$ This clearly shows that such a low $Q$ (X954 ray or neutron scattering) peak feature appears at any 955 concentration conditions (similar to what was reported by 956 Zhang et al. ${ }^{28}$ and by Liu et al., ${ }^{55}$ but at odds with what Tan et $957 \mathrm{al}^{22}$ claimed). Such a low $Q$ feature then represents the 958 signature of a structural organization that is persistent in the 959 WiS system over the whole concentration regime. At low salt 960 content, it clearly reflects the existence of the globular 961 aggregates dispersed in water. When the salt content increases, 962 the peak fingerprints the existence of a distinctly bicontinuous, 963 sponge-like morphology, with mutually excluding domains 964 formed by the more extended phase (water or, at high salt 965 content, anions), which alternates, over nm scale, with the 966 minority one (anions or, at high salt content, water). At 967 concentration extremes (either water-rich or salt-rich con968 ditions), the majority phase constitutes a percolating network 969 hold up either by hydrogen-bonding correlations between 970 water molecules (water-rich case) or by cation/anion and 971 fluorophilic dispersive correlations (salt-rich case). These 972 dominating matrixes are intercalated either by anion globules 973 (water-rich case) or by water-lithium wires (salt-rich case) 974 with extensions of $10-30 \AA$, but not percolating.

975 To obtain further experimental evidence about intermolec976 ular interactions existing in the LiIM14-water system, 977 vibrational modes of water were analyzed by means of FTIR 978 and Raman spectroscopy. Indeed, the water stretching and bending modes are known to be powerful probes for 979 monitoring the strength and configuration of the H-bond 980 network. $^{86}$ Selected portions of FTIR and Raman spectra for 981 six LiIM14- $\mathrm{H}_{2} \mathrm{O}$ mixtures at $c=1,2,5,10,15$, and $20 \mathrm{~m}$ are 982 shown in Figure 11a,b, respectively. The water stretching and 983 f11
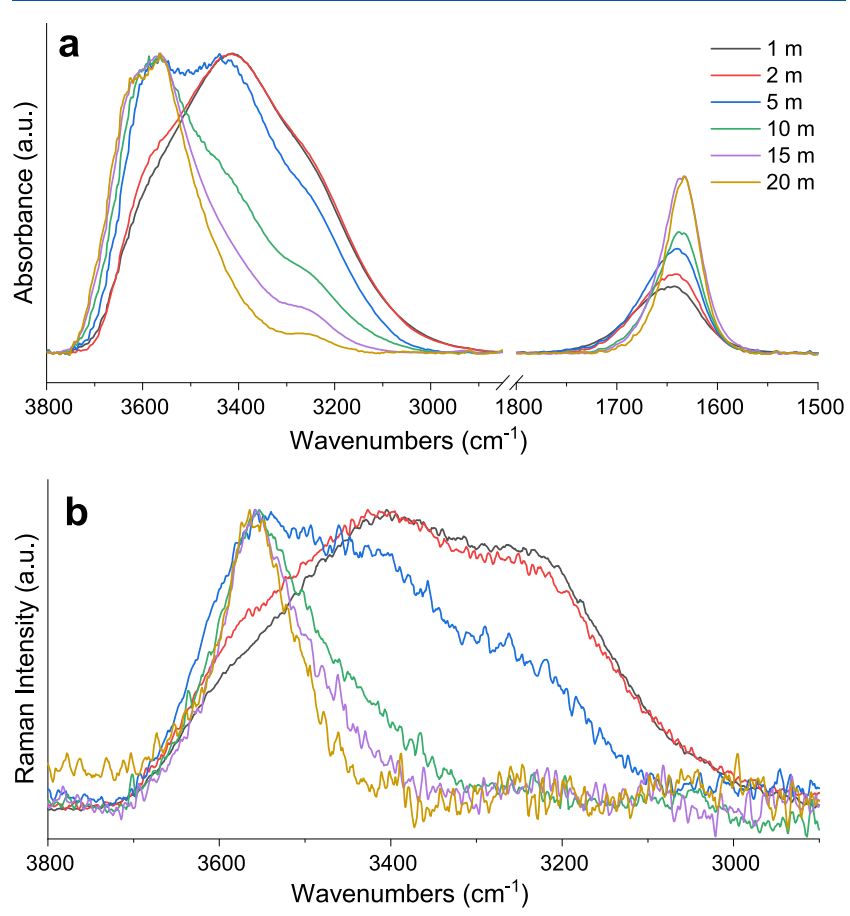

Figure 11. FTIR spectra (a) and Raman spectra (b) acquired at room temperature for LiIM14- $\mathrm{H}_{2} \mathrm{O}$ mixtures at different molalities $(m)$. In (b), wavenumbers of the $x$-axis identify Raman shifts.

bending region for FTIR spectroscopy (3800-2850 and 984 1850-1450 $\mathrm{cm}^{-1}$, respectively), as well as the stretching 985 region for Raman spectroscopy $\left(3800-2800 \mathrm{~cm}^{-1}\right)$, have been 986 analyzed by means of the MCR-ALS model (see Methods). 987 For both the acquired data sets, the spectral evolution as a 988 function of salt concentration has been modeled in terms of a 989 linear combination of three spectral components, each one 990 assigned to water populations with different degrees of 991 intermolecular interactions. In analogy to other spectral 992 decomposition techniques applied to similar systems, ${ }^{28}$ the 993 three spectral profiles for the FTIR and Raman are shown in 994 Figure 12a,b, respectively. For both FTIR and Raman spectral 995 f12 profile sets, a first component, defined as $2 \mathrm{w}$, predominates at 996 the lowest salt concentration (blue lines in Figure 12a,b). In 997 both cases, a great similarity to neat water spectra is observed; 998 these spectral profiles, indeed, are assigned to a bulk-water 999 population, where each water molecule donates on average two 1000 $\mathrm{H}$-bond to other water molecules. In particular, in the $\mathrm{OH} 1001$ stretching spectral region, the FTIR absorption shows a broad 1002 band centered at $3422 \mathrm{~cm}^{-1}$, whereas in the Raman profile, 1003 three main spectral contributions are distinguishable although 1004 overlapped. They are centered at 3560, 3450, and $3260 \mathrm{~cm}^{-1} 1005$ and assigned to the $\mathrm{OH}$ vibrations of water molecules with an 1006 increasing degree of connectivity, respectively. ${ }^{87}$ On the other 1007 hand, a second spectral profile, indicated as 2a (red lines in 1008 Figure 12a,b), predominates at high LiIM14 concentrations; it 1009 is assigned to water molecules that donate two H-bonds to 1010 other molecular species, i.e., the IM14 anion. In fact, for both 1011 the FTIR and the Raman case, it shows the characteristic 1012 

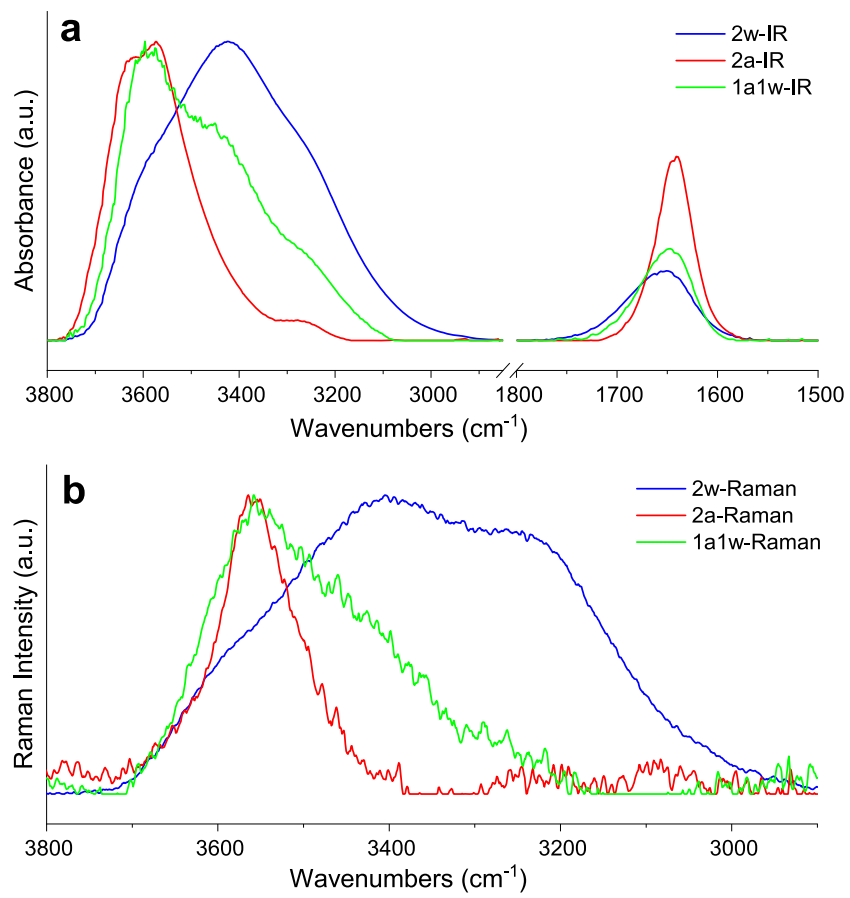

Figure 12. Spectral profiles of the $2 \mathrm{w}$ (blue line), 2a (red line), and lalw (green line) water populations calculated by MCR-ALS for the (a) FTIR and (b) Raman data sets. In (b), the wavenumbers of the $x$ axis identify the Raman shifts.

1013 spectral features of isolated water in the solution, such as the 1014 blue-shifted $\mathrm{OH}$ stretching, with its asymmetric and symmetric 1015 intramolecular coupling modes falling at 3640 and $3570 \mathrm{~cm}^{-1}$, 1016 respectively. ${ }^{88}$ Finally, a third spectral component, defined as 1017 1wla (green lines in Figure 12a,b), is assigned to those water 1018 molecules donating on average one H-bond to water; 1019 meanwhile, the other is weakly bonded to the IM14 anion. 1020 Spectral features, such as bandwidths and positions of the $1 \mathrm{a} 1 \mathrm{w}$ 1021 spectrum, are intermediate between the $2 \mathrm{a}$ and $2 \mathrm{w}$ 1022 components. Moreover, in the FTIR spectral profiles, the $1023 \mathrm{OH}$ bending mode at $1652 \mathrm{~cm}^{-1}$ is clearly observable. It is 1024 broad in the $2 \mathrm{w}$-IR component, with a full width at half 1025 maximum $(\mathrm{FWHM})=88 \mathrm{~cm}^{-1}$ and red shifts and distinctly 1026 sharpens (down to a FWHM $=45 \mathrm{~cm}^{-1}$, in the 2a-IR) with 1027 increasing salt concentration. This sharpening is additional 1028 evidence of the lack of the H-bonded water network, due to 1029 the absence of the intermolecular mode coupling between the 1030 pure $\mathrm{H}-\mathrm{O}-\mathrm{H}$ bending and the libration mode, typical of 1031 tetrahedral water clusters. ${ }^{89}$

1032 The application of the MCR-ALS algorithm to the modeling 1033 of experimental FTIR and Raman spectroscopy data delivers 1034 the concentration dependence of the three components $2 \mathrm{w}, 2 \mathrm{a}$, 1035 and $1 w 1 a$, i.e., the weights of the three water populations $2 a$, $10362 \mathrm{w}$, and $1 \mathrm{alw}$ in building up the observed spectra. Figure 13 1037 displays these relative weights (sum of the three species 1038 populations normalized to one), independently calculated for 1039 IR and Raman results, as a function of salt concentration. It is 1040 noteworthy that, although the two complementary vibrational 1041 techniques, FTIR and Raman, experimentally deliver different 1042 spectra (Figure 11), the MCR-ALS calculated concentration 1043 profiles (Figure 12) show very consistent trends (Figure 13). 1044 This strongly supports the reliability of the analysis and 1045 prompts for the following interpretative model.

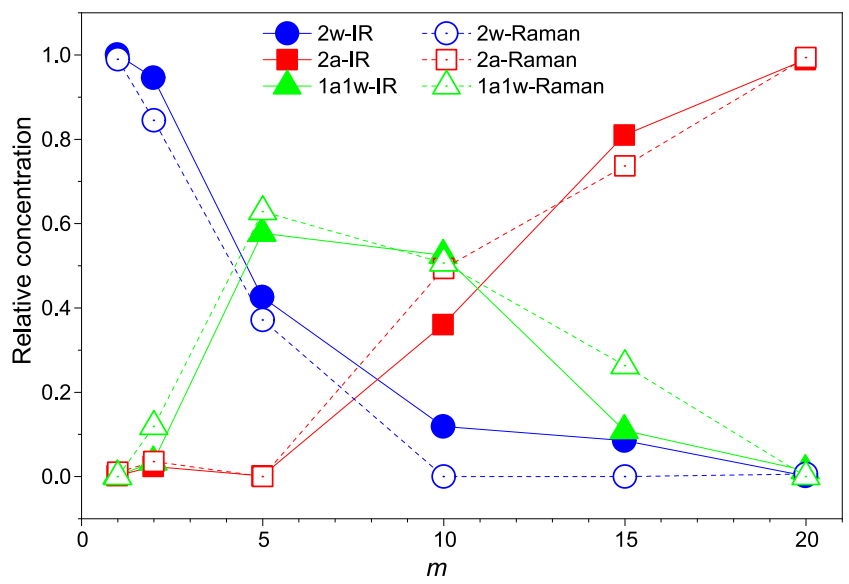

Figure 13. Concentration profiles of the $2 \mathrm{w}$ (blue circles), 2a (red squares), and $1 \mathrm{a} 1 \mathrm{w}$ (green triangles) water populations calculated by MCR-ALS for the FTIR (filled symbols) and Raman (open symbols) spectral data sets.

At low salt content, e.g., $c<5 \mathrm{~m}$, water is mostly in the $2 \mathrm{w} 1046$ form with a small population of lalw due to the large 1047 abundance of bulk water with respect to the solvation shell of 1048 the salt ions (Figure 9a,b). At $c=5 \mathrm{~m}$, a distinct increase of the 1049 lalw water population is observed, up to about $60 \%$ of the 1050 total, at the expense of $2 \mathrm{w}$ water; meanwhile, the $2 \mathrm{a}$ water is 1051 still absent (Figure 9c). An onset of a structural transition from 1052 a water continuous matrix to a bicontinuous water-LiIM14 1053 phase is observed at about $c=7 \mathrm{~m}$ (Figure 9d). At this 1054 concentration, most water molecules donate one H-bond to an 1055 adjacent water molecule and the other to the IM14 anion, but 1056 still, water clusters survive, contributing to the $2 \mathrm{w}$ concen- 1057 tration.

1058

By further increasing the salt concentration $(c \geq 10 \mathrm{~m})$, the 1059 $2 \mathrm{w}$ water population becomes irrelevant; meanwhile, a large 1060 increase of the 2a population is highlighted up to almost $100 \% 1061$ at $c=20 \mathrm{~m}$, at the expense of the $1 \mathrm{alw}$ water. This trend 1062 agrees with the findings from MD simulations: upon increasing 1063 the salt content, water molecules organize into progressively 1064 smaller clusters/chains in the anion matrix. At $c=20 \mathrm{~m}$, only a 1065 very few water molecules interact with each other, although the 1066 average distance between their mass center is $3.2 \AA$ (Figure 1067 $6 \mathrm{a})$. They are involved in hydrogen bonds with the anion and 1068 coordinated around the cation (Figure 8). This behavior is 1069 different from the one observed in the case of the smaller anion 1070 TFSI, where a 50\% population of 1 wla coordination managed 1071 to survive at $\mathcal{c}_{\text {TFSI }}=20 \mathrm{~m} ;{ }^{28}$ here, presumably due to the much 1072 larger hydrophobic portion, the anion network tends to 1073 strongly separate water molecules from each other.

\section{CONCLUSIONS}

1074

Water-in-salt systems are attracting great attention as appealing 1076 electrolytes for energy storage devices. While several WiS have 1077 been proposed aiming at extending the electrochemical and 1078 liquid state stability, nevertheless, structural investigations have 1079 focused mostly on LiTFSI-based WiS systems. Here, we 1080 reported the first investigation on the phase diagram, 1081 electrochemical properties, structure, and vibrational features 1082 of a novel lithium electrolyte based on the 1083 ((trifluoromethane)(nonafluorobutane)-sulfonyl)imide anion, 1084 a highly asymmetric ionic species. This electrolyte class shows 1085 appealing liquid and electrochemical stability windows, with $c 1086$ 
$1087=20 \mathrm{~m}$ mixtures melting at $<25{ }^{\circ} \mathrm{C}$, and interesting 1088 conductivity performances. The synergic exploitation of IR 1089 and Raman spectroscopies together with X-ray small- and 1090 wide-angle scattering and molecular dynamics simulations 1091 allows achieving a very detailed insight into the structural 1092 features of such a system. The liquid state is characterized by a 1093 strong segregation between water and hydrophobic fluorous 1094 ionic moieties: the large fluorous tails enhance such a 1095 segregation as compared, e.g., to the well-known LiTFSI1096 water systems. In this scenario, dilute solutions are 1097 characterized by a peculiar globular organization of anions 1098 that are immersed into homogenous bulk-like water. This 1099 behavior manifests itself as a very strong X-ray scattering 1100 feature, whose presence is ubiquitous across the explored 1101 concentration range. Ionic species are fully water solvated, and 1102 no ion pairing can be observed. Upon increasing the salt 1103 content, lithium keeps on drawing water molecules but begins 1104 interacting with the anions by maintaining a constant 1105 coordination number of oxygen atoms (either from water or 1106 from the anions) over the whole concentration range. Anions 1107 tend to be locally neutralized by (water-bearing) lithium ions; 1108 otherwise, they interact either with water (via HB interactions) 1109 or between themselves through dispersive, fluorophilic 1110 interactions, leading to a progressively more and more 1111 extended and percolating fluorous matrix. At the highest 1112 concentration conditions, the large anion size leads to an 1113 extreme fragmentation of a bulk-water hydrogen-bonding 1114 network and no more water-water correlations can be 1115 detected. Water then organizes in a wire-like manner with a 1116 negligible amount of water-water hydrogen-bonding-mediated 1117 correlations, but with intermediate lithium ions mediating the 1118 correlation and locally neutralizing the system. Overall, the 1119 present scenario supports and, considering the different anions, 1120 reinforces the proposal that no water channels exist at high salt 1121 content. The fluorous matrix percolates across the bulk and is 1122 locally intercalated by short water-lithium wires, across which, 1123 presumably, lithium hopping occurs. Accordingly, lithium's 1124 peculiar transport properties in WiS systems seem to be related 1125 to two concomitant effects, namely: (i) the extreme nanoscale 1126 separation between anion-rich and water-rich domains that 1127 appear ubiquitous across the probed concentration range and 1128 (ii) the existence of water wires across the anion matrix where 1129 a large population of $\mathrm{Li}\left(\mathrm{H}_{2} \mathrm{O}\right)_{4}{ }^{+}$clusters, fully disentangled 1130 from anion coordination, would diffuse at an enhanced rate, as 1131 compared to anion-bound lithium.

1132 Furthermore, we stress that while these preliminary 1133 characterizations indicate that the anodic stability of the 1134 LiIM14 electrolyte does not match the requirements for high 1135 voltage $(>4 \mathrm{~V})$ battery systems, this electrolyte still represents 1136 a new, appealing way for enhancing the electrochemical 1137 robustness of aqueous solutions toward oxidation. For 1138 instance, concentrated aqueous electrolytes based on the 1139 IM14 anion might find applications in metal-oxygen batteries 1140 (i.e., $\mathrm{Zn} / \mathrm{O}_{2}$ systems operate below $2 \mathrm{~V}$ ) and, upon an 1141 enhancement of the anodic stability of even a few hundreds of $1142 \mathrm{mV}$, in $\mathrm{Li} / \mathrm{S}$ post-lithium batteries.

1143 The present study provides a detailed characterization of the 1144 organization in an evolved WiS (as compared to TFSI based 1145 ones), suggesting a role for strong anion asymmetry in 1146 structural organization and thus prompting for knowledge1147 oriented modifications to be applied to the existing WiS 1148 systems.

\section{ASSOCIATED CONTENT}

(s) Supporting Information

1150

The Supporting Information is available free of charge at 1151 https://pubs.acs.org/doi/10.1021/acs.jpcb.1c06759. 1152

Supporting figures on the density of LiIM14- $\mathrm{H}_{2} \mathrm{O} 1153$ mixtures as a function of temperature; log-log version 1154 of SAXS data; computed neutron scattering patterns; 1155 comparison between the experimental and computed 1156 density at $25{ }^{\circ} \mathrm{C}$; MD-derived running coordination 1157 numbers from $g(r)$ 's; several solvation distribution 1158 functions and corresponding average coordination 1159 numbers (PDF)

1160

\section{AUTHOR INFORMATION}

1161

Corresponding Authors

Alessandro Triolo - Laboratorio Liquidi Ionici, Istituto 1163 Struttura della Materia, Consiglio Nazionale delle Ricerche 1164 (ISM-CNR), Rome 00133, Italy; ㅇoㅇid.org/0000-0003- 1165 4074-0743; Email: triolo@ism.cnr.it

1166

Olga Russina - Laboratorio Liquidi Ionici, Istituto Struttura 1167 della Materia, Consiglio Nazionale delle Ricerche (ISM- 1168 CNR), Rome 00133, Italy; Department of Chemistry, 1169 University of Rome Sapienza, Rome 00185, Italy; 1170 Email: olga.russina@uniroma1.it

1170
1171

Authors

1172

Valerio Di Lisio - Department of Chemistry, University of 1173 Rome Sapienza, Rome 00185, Italy

Fabrizio Lo Celso - Laboratorio Liquidi Ionici, Istituto 1175 Struttura della Materia, Consiglio Nazionale delle Ricerche 1176 (ISM-CNR), Rome 00133, Italy; Department of Physics and 1177 Chemistry, Università di Palermo, Palermo 90133, Italy 1178

Giovanni B. Appetecchi - ENEA, SSPT-PROMAS- 1179 MATPRO Technical Unit, Rome 00123, Italy; (1) orcid.org/ 1180 0000-0002-6623-0373

Barbara Fazio - Istituto Processi Chimico-Fisici, Consiglio 1182 Nazionale delle Ricerche (IPCF-CNR), Messina 98158, 1183 Italy; (1) orcid.org/0000-0002-1947-1123

Philip Chater - Diamond House, Harwell Science \& Innovation Campus, Diamond Light Source, Ltd., Didcot 1186 OX11 ODE, U.K.; (1) orcid.org/0000-0002-5513-9400 1187

Andrea Martinelli - Department of Chemistry, University of 1188 Rome Sapienza, Rome 00185, Italy; (1) orcid.org/0000- 1189 0002-6401-9988

1190

Fabio Sciubba - Department of Chemistry, University of 1191 Rome Sapienza, Rome 00185, Italy; NMR-Based 1192 Metabolomics Laboratory (NMLab), Sapienza University of 1193 Rome, Rome 00185, Italy; (1) orcid.org/0000-0002-1982- 1194 2712

Complete contact information is available at:

https://pubs.acs.org/10.1021/acs.jpcb.1c06759

Notes

The authors declare no competing financial interest.

\section{ACKNOWLEDGMENTS}

This work has been supported by the University of Rome 1201 Sapienza Projects: RG11715C7CC660BE and 1202 RM120172B2165468. The authors thank Diamond Light 1203 Source for access to beamline I15-1 (CY27222-1). Access to 1204 the SAXS-Lab at the University of Rome Sapienza and support 1205 from Dr. A. Del Giudice are acknowledged. 
1208 (1) Suo, L.; Borodin, O.; Gao, T.; Olguin, M.; Ho, J.; Fan, X.; Luo, 1209 C.; Wang, C.; Xu, K. Water-in-Salt” Electrolyte Enables High-Voltage 1210 Aqueous Lithium-Ion Chemistries. Science 2015, 350, 938-943.

1211 (2) Liang, T.; Hou, R.; Dou, Q.; Zhang, H.; Yan, X. The 1212 Applications of Water-in-Salt Electrolytes in Electrochemical Energy 1213 Storage Devices. Adv. Funct. Mater. 2021, 31, No. 2006749.

1214 (3) Yamada, Y.; Wang, J.; Ko, S.; Watanabe, E.; Yamada, A. 1215 Advances and Issues in Developing Salt-Concentrated Battery 1216 Electrolytes. Nat. Energy 2019, 4, 269-280.

1217 (4) Azov, V. A.; Egorova, K. S.; Seitkalieva, M. M.; Kashin, A. S.; 1218 Ananikov, V. P. "Solvent-in-Salt” Systems for Design of New Materials 1219 in Chemistry, Biology and Energy Research. Chem. Soc. Rev. 2018, 47, 1220 1250-1284.

1221 (5) Zheng, Q.; Miura, S.; Miyazaki, K.; Ko, S.; Watanabe, E.; 1222 Okoshi, M.; Chou, C. P.; Nishimura, Y.; Nakai, H.; Kamiya, T.; 1223 Honda, T.; Akikusa, J.; Yamada, Y.; Yamada, A. Sodium- and 1224 Potassium-Hydrate Melts Containing Asymmetric Imide Anions for 1225 High-Voltage Aqueous Batteries. Angew. Chem., Int. Ed. 2019, 58, $122614202-14207$.

1227 (6) Becker, M.; Kühnel, R. S.; Battaglia, C. Water-in-Salt Electrolytes 1228 for Aqueous Lithium-Ion Batteries with Liquidus Temperatures below $1229-10{ }^{\circ}$ c. Chem. Commun. 2019, 55, 12032-12035.

1230 (7) Reber, D.; Kühnel, R. S.; Battaglia, C. High-Voltage Aqueous 1231 Supercapacitors Based on NaTFSI. Sustainable Energy Fuels 2017, 1, 1232 2155-2161.

1233 (8) Amiri, M.; Bélanger, D. Physicochemical and Electrochemical 1234 Properties of Water-in-Salt Electrolytes. ChemSusChem 2021, 14, 1235 2487-2500.

1236 (9) Ko, S.; Yamada, Y.; Miyazaki, K.; Shimada, T.; Watanabe, E.; 1237 Tateyama, Y.; Kamiya, T.; Honda, T.; Akikusa, J.; Yamada, A. 1238 Lithium-Salt Monohydrate Melt: A Stable Electrolyte for Aqueous 1239 Lithium-Ion Batteries. Electrochem. Commun. 2019, 104, No. 106488. 1240 (10) Dubouis, N.; Lemaire, P.; Mirvaux, B.; Salager, E.; Deschamps, 1241 M.; Grimaud, A. The Role of the Hydrogen Evolution Reaction in the 1242 Solid-Electrolyte Interphase Formation Mechanism for "Water-in1243 Salt” Electrolytes. Energy Environ. Sci. 2018, 11, 3491-3499.

1244 (11) Shen, Y.; Liu, B.; Liu, X.; Liu, J.; Ding, J.; Zhong, C.; Hu, W. 1245 Water-in-Salt Electrolyte for Safe and High-Energy Aqueous Battery. 1246 Energy Storage Mater. 2021, 34, 461-474.

1247 (12) Yamada, Y.; Usui, K.; Sodeyama, K.; Ko, S.; Tateyama, Y.; 1248 Yamada, A. Hydrate-Melt Electrolytes for High-Energy-Density 1249 Aqueous Batteries. Nat. Energy 2016, 1, No. 16129.

1250 (13) Borodin, O.; Suo, L.; Gobet, M.; Ren, X.; Wang, F.; Faraone, 1251 A.; Peng, J.; Olguin, M.; Schroeder, M.; Ding, M. S.; Gobrogge, E.; 1252 von Wald Cresce, A.; Munoz, S.; Dura, J. A.; Greenbaum, S.; Wang, 1253 C.; Xu, K. Liquid Structure with Nano-Heterogeneity Promotes 1254 Cationic Transport in Concentrated Electrolytes. ACS Nano 2017, 11, 1255 10462-10471.

1256 (14) Suo, L.; Borodin, O.; Wang, Y.; Rong, X.; Sun, W.; Fan, X.; Xu, 1257 S.; Schroeder, M. A.; Cresce, A. V.; Wang, F.; Yang, C.; Hu, Y. S.; Xu, 1258 K.; Wang, C. Water-in-Salt” Electrolyte Makes Aqueous Sodium-Ion 1259 Battery Safe, Green, and Long-Lasting. Adv. Energy Mater. 2017, 7, 1260 No. 1701189.

1261 (15) Chen, M.; Feng, G.; Qiao, R. Water-in-Salt Electrolytes: An 1262 Interfacial Perspective. Curr. Opin. Colloid Interface Sci. 2020, 47, 991263110.

1264 (16) Li, H.; Kurihara, T.; Yang, D.; Watanabe, M.; Ishihara, T. A 1265 Novel Aqueous Dual-Ion Battery Using Concentrated Bisalt Electro1266 lyte. Energy Storage Mater. 2021, 38, 454-461.

1267 (17) Miyazaki, K.; Takenaka, N.; Watanabe, E.; Iizuka, S.; Yamada, 1268 Y.; Tateyama, Y.; Yamada, A. First-Principles Study on the Peculiar 1269 Water Environment in a Hydrate-Melt Electrolyte. J. Phys. Chem. Lett. 1270 2019, 10, 6301-6305.

1271 (18) Ding, M. S.; Xu, K. Phase Diagram, Conductivity, and Glass 1272 Transition of LiTFSI-H2O Binary Electrolytes. J. Phys. Chem. C 2018, 1273 122, 16624-16629.
(19) Ding, M. S.; Von Cresce, A.; Xu, K. Conductivity, Viscosity, 1274 and Their Correlation of a Super-Concentrated Aqueous Electrolyte. 1275 J. Phys. Chem. C 2017, 121, 2149-2153.

1276

(20) Sakamoto, R.; Yamashita, M.; Nakamoto, K.; Zhou, Y.; 1277 Yoshimoto, N.; Fujii, K.; Yamaguchi, T.; Kitajou, A.; Okada, S. Local 1278 Structure of a Highly Concentrated NaClO4aqueous Solution-Type 1279 Electrolyte for Sodium Ion Batteries. Phys. Chem. Chem. Phys. 2020, 1280 22, 26452-26458.

1281

(21) Lim, J.; Park, K.; Lee, H.; Kim, J.; Kwak, K.; Cho, M. 1282 Nanometric Water Channels in Water-in-Salt Lithium Ion Battery 1283 Electrolyte. J. Am. Chem. Soc. 2018, 140, 15661-15667.

(22) Tan, P.; Yue, J.; Yu, Y.; Liu, B.; Liu, T.; Zheng, L.; He, L.; 1285 Zhang, X.; Suo, L.; Hong, L. Solid-Like Nano-Anion Cluster 1286 Constructs a Free Lithium-Ion-Conducting Superfluid Framework 1287 in a Water-in-Salt Electrolyte. J. Phys. Chem. C 2021, 125, 11838- 1288 11847.

1289

(23) González, M. A.; Borodin, O.; Kofu, M.; Shibata, K.; Yamada, 1290 T.; Yamamuro, O.; Xu, K.; Price, D. L.; Saboungi, M.-L. Nanoscale 1291 Relaxation in "Water-in-Salt" and "Water-in-Bisalt" Electrolytes. J. 1292 Phys. Chem. Lett. 2020, 7279-7284.

1293

(24) Popov, I.; Sacci, R. L.; Sanders, N. C.; Matsumoto, R. A.; 1294 Thompson, M. W.; Osti, N. C.; Kobayashi, T.; Tyagi, M.; Mamontov, 1295 E.; Pruski, M.; Cummings, P. T.; Sokolov, A. P. Critical Role of 1296 Anion-Solvent Interactions for Dynamics of Solvent-in-Salt Solutions. 1297 J. Phys. Chem. C 2020, 124, 8457-8466.

1298

(25) Zhang, M.; Hao, H.; Zhou, D.; Duan, Y.; Wang, Y.; Bian, H. 1299 Understanding the Microscopic Structure of a "Water-in-Salt" 1300 Lithium Ion Battery Electrolyte Probed with Ultrafast IR Spectros- 1301 copy. J. Phys. Chem. C 2020, 124, 8594-8604.

1302

(26) Reber, D.; Takenaka, N.; Kühnel, R. S.; Yamada, A.; Battaglia, 1303 C. Impact of Anion Asymmetry on Local Structure and Supercooling 1304 Behavior of Water-in-Salt Electrolytes. J. Phys. Chem. Lett. 2020, 11, 1305 $4720-4725$.

1306

(27) Jeon, J.; Lee, H.; Choi, J. H.; Cho, M. Modeling and Simulation 1307 of Concentrated Aqueous Solutions of LiTFSI for Battery 1308 Applications. J. Phys. Chem. C 2020, 124, 11790-11799. 1309

(28) Zhang, Y.; Lewis, N. H. C.; Mars, J.; Wan, G.; Weadock, N. J.; 1310 Takacs, C. J.; Lukatskaya, M. R.; Steinrück, H.-G.; Toney, M. F.; 1311 Tokmakoff, A.; Maginn, E. J. Water-in-Salt LiTFSI Aqueous 1312 Electrolytes. 1. Liquid Structure from Combined Molecular Dynamics 1313 Simulation and Experimental Studies. J. Phys. Chem. B 2021, 125, 1314 4501-4513.

1315

(29) Biswas, A.; Mallik, B. S. Ultrafast Aqueous Dynamics in 1316 Concentrated Electrolytic Solutions of Lithium Salt and Ionic Liquid. 1317 J. Phys. Chem. B 2020, 124, 9898-9912.

1318

(30) Han, K. S.; Yu, Z.; Wang, H.; Redfern, P. C.; Ma, L.; Cheng, L.; 1319 Chen, Y.; Hu, J. Z.; Curtiss, L. A.; Xu, K.; Murugesan, V.; Mueller, K. 1320 T. Origin of Unusual Acidity and Li+Diffusivity in a Series of Water- 1321 in-Salt Electrolytes. J. Phys. Chem. B 2020, 124, 5284-5291. 1322

(31) Mendez-Morales, T.; Li, Z.; Salanne, M. Computational 1323 Screening of the Physical Properties of Water-in-Salt Electrolytes. 1324 Batteries Supercaps 2021, 4, 646-652.

1325

(32) Borodin, O.; Self, J.; Persson, K. A.; Wang, C.; Xu, K. 1326 Uncharted Waters: Super-Concentrated Electrolytes. Joule 2020, 4, 1327 69-100.

1328

(33) Yu, Z.; Curtiss, L. A.; Winans, R. E.; Zhang, Y.; Li, T.; Cheng, 1329 L. Asymmetric Composition of Ionic Aggregates and the Origin of 1330 High Correlated Transference Number in Water-in-Salt Electrolytes. 1331 J. Phys. Chem. Lett. 2020, 11, 1276-1281.

1332

(34) Forero-Saboya, J.; Hosseini-Bab-Anari, E.; Abdelhamid, M. E.; 1333 Moth-Poulsen, K.; Johansson, P. Water-in-Bisalt Electrolyte with 1334 Record Salt Concentration and Widened Electrochemical Stability 1335 Window. J. Phys. Chem. Lett. 2019, 10, 4942-4946.

1336

(35) Tsurumura, T.; Hashimoto, Y.; Morita, M.; Umebayashi, Y.; 1337 Fujii, K. Anion Coordination Characteristics of Ion-Pair Complexes in 1338 Highly Concentrated Aqueous Lithium Bis-1339 (Trifluoromethanesulfonyl) Amide Electrolytes. Anal. Sci. 2019, 35, 1340 289-294. 
1342 (36) Horwitz, G.; Rodríguez, C. R.; Steinberg, P. Y.; Burton, G.; 1343 Corti, H. R. Mobility-Viscosity Decoupling and Cation Transport in 1344 Water-in-Salt Lithium Electrolytes. Electrochim. Acta 2020, 359, 1345 No. 136915.

1346 (37) Kühnel, R.-S.; Reber, D.; Battaglia, C. Perspective-Electro1347 chemical Stability of Water-in-Salt Electrolytes. J. Electrochem. Soc. 1348 2020, 167, No. 070544.

1349 (38) Droguet, L.; Grimaud, A.; Fontaine, O.; Tarascon, J. M. Water1350 in-Salt Electrolyte (WiSE) for Aqueous Batteries: A Long Way to 1351 Practicality. Adv. Energy Mater. 2020, 10, No. 2002440.

1352 (39) Tan, J.; Liu, J. Electrolyte Engineering Toward High-Voltage 1353 Aqueous Energy Storage Devices. Energy Environ. Mater. 2020, 4, 1354 302-306.

1355 (40) Reber, D.; Grissa, R.; Becker, M.; Kühnel, R.; Battaglia, C. 1356 Anion Selection Criteria for Water-in-Salt Electrolytes. Adv. Energy 1357 Mater. 2021, 11, No. 2002913.

1358 (41) von Wald Cresce, A.; Xu, K. Aqueous Lithium-ion Batteries. 1359 Carbon Energy 2021, 3, 721-751.

1360 (42) Watanabe, H.; Arai, N.; Nozaki, E.; Han, J.; Fujii, K.; Ikeda, K.; 1361 Otomo, T.; Ueno, K.; Dokko, K.; Watanabe, M.; Kameda, Y.; 1362 Umebayashi, Y. Local Structure of $\mathrm{Li}+$ in Superconcentrated 1363 Aqueous LiTFSA Solutions. J. Phys. Chem. B 2021, 125, 7477-7484. 1364 (43) Marcus, Y. Unconventional Deep Eutectic Solvents: Aqueous 1365 Salt Hydrates. ACS Sustainable Chem. Eng. 2017, 5, 11780-11787. 1366 (44) Lux, S. F.; Terborg, L.; Hachmöller, O.; Placke, T.; Meyer, H.1367 W.; Passerini, S.; Winter, M.; Nowak, S. LiTFSI Stability in Water and 1368 Its Possible Use in Aqueous Lithium-Ion Batteries: PH Dependency, 1369 Electrochemical Window and Temperature Stability. J. Electrochem. 1370 Soc. 2013, 160, A1694-A1700.

1371 (45) Reber, D.; Kühnel, R. S.; Battaglia, C. Suppressing 1372 Crystallization of Water-in-Salt Electrolytes by Asymmetric Anions 1373 Enables Low-Temperature Operation of High-Voltage Aqueous 1374 Batteries. ACS Mater. Lett. 2019, 1, 44-51.

1375 (46) Ko, S.; Yamada, Y.; Yamada, A. A $62 \mathrm{~m}$ K-Ion Aqueous 1376 Electrolyte. Electrochem. Commun. 2020, 116, No. 106764.

1377 (47) Thareja, S.; Kumar, A. "water-In-Salt" Electrolyte-Based High1378 Voltage $(2.7$ V) Sustainable Symmetric Supercapacitor with Superb 1379 Electrochemical Performance - An Analysis of the Role of Electrolytic 1380 Ions in Extending the Cell Voltage. ACS Sustainable Chem. Eng. 2021, 1381 9, 2338-2347.

1382 (48) Horwitz, G.; Härk, E.; Steinberg, P. Y.; Cavalcanti, L. P.; Risse, 1383 S.; Corti, H. R. The Nanostructure of Water-in-Salt Electrolytes 1384 Revisited: Effect of the Anion Size. ACS Nano 2021, 15, 11564138511572.

1386 (49) Horwitz, G.; Steinberg, P. Y.; Corti, H. R. Volumetric and 1387 Viscosity Properties of Water-in-Salt Lithium Electrolytes: A 1388 Comparison with Ionic Liquids and Hydrated Molten Salts. J. 1389 Chem. Thermodyn. 2021, 158, No. 106457.

1390 (50) Russina, O.; Triolo, A. New Experimental Evidence Supporting 1391 the Mesoscopic Segregation Model in Room Temperature Ionic 1392 Liquids. Faraday Discuss. 2012, 154, 97-109.

1393 (51) Lo Celso, F.; Appetecchi, G. B.; Simonetti, E.; Zhao, M.; Jr, E. 1394 W. C.; Keiderling, U.; Gontrani, L.; Triolo, A. Microscopic Structural 1395 and Dynamic Features in Triphilic Room Temperature Ionic Liquids. 1396 Front. Chem. 2019, 7, No. 285.

1397 (52) Lo Celso, F.; Appetecchi, G. B.; Jafta, C. J.; Gontrani, L.; 1398 Canongia Lopes, J. N.; Triolo, A.; Russina, O. Nanoscale 1399 Organization in the Fluorinated Room Temperature Ionic Liquid: 1400 Tetraethyl Ammonium (Trifluoromethanesulfonyl)1401 (Nonafluorobutylsulfonyl)Imide. J. Chem. Phys. 2018, 148, 1402 No. 193816.

1403 (53) Lo Celso, F.; Appetecchi, G. B.; Simonetti, E.; Keiderling, U.; 1404 Gontrani, L.; Triolo, A.; Russina, O. Mesoscopic Structural 1405 Organization in Fluorinated Pyrrolidinium-Based Room Temperature 1406 Ionic Liquids. J. Mol. Liq. 2019, 289, No. 111110.

1407 (54) Triolo, A.; Lo Celso, F.; Ottaviani, C.; Ji, P.; Appetecchi, G. B.; 1408 Leonelli, F.; Keeble, D. S.; Russina, O. Structural Features of Selected 1409 Protic Ionic Liquids Based on a Super-Strong Base. Phys. Chem. Chem. 1410 Phys. 2019, 21, 25369-25378.
(55) Liu, X.; Yu, Z.; Sarnello, E.; Qian, K.; Seifert, S.; Winans, R. E.; 1411 Cheng, L.; Li, T. Microscopic Understanding of the Ionic Networks of 1412 "Water-in-Salt" Electrolytes. Energy Mater. Adv. 2021, 2021, 1413 No. 7368420.

(56) Filik, J.; Ashton, A. W.; Chang, P. C. Y.; Chater, P. A.; Day, S. 1415 J.; Drakopoulos, M.; Gerring, M. W.; Hart, M. L.; Magdysyuk, O. V.; 1416 Michalik, S.; Smith, A.; Tang, C. C.; Terrill, N. J.; Wharmby, M. T.; 1417 Wilhelm, H. Processing Two-Dimensional X-Ray Diffraction and 1418 Small-Angle Scattering Data in DAWN 2. J. Appl. Crystallogr. 2017, 1419 50, 959-966.

1420

(57) Jaumot, J.; de Juan, A.; Tauler, R. MCR-ALS GUI 2.0: New 1421 Features and Applications. Chemom. Intell. Lab. Syst. 2015, 140, 1-12. 1422 (58) Garrido, M.; Rius, F. X.; Larrechi, M. S. Multivariate Curve 1423 Resolution-Alternating Least Squares (MCR-ALS) Applied to 1424 Spectroscopic Data from Monitoring Chemical Reactions Processes. 1425 Anal. Bioanal. Chem. 2008, 390, 2059-2066.

1426

(59) Ahmadi, R.; Hemmateenejad, B.; Safavi, A.; Shojaeifard, Z.; 1427 Shahsavar, A.; Mohajeri, A.; Heydari Dokoohaki, M.; Zolghadr, A. R. 1428 Deep Eutectic-Water Binary Solvent Associations Investigated by 1429 Vibrational Spectroscopy and Chemometrics. Phys. Chem. Chem. Phys. 1430 2018, 20, 18463-18473.

1431

(60) Hess, B.; Kutzner, C.; van der Spoel, D.; Lindahl, E. 1432 GROMACS 4: Algorithms for Highly Efficient, Load-Balanced, and 1433 Scalable Molecular Simulation. J. Chem. Theory Comput. 2008, 4, 1434 435-447.

1435

(61) Van Der Spoel, D.; Lindahl, E.; Hess, B.; Groenhof, G.; Mark, 1436 A. E.; Berendsen, H. J. C. GROMACS: Fast, Flexible, and Free. J. 1437 Comput. Chem. 2005, 26, 1701-1718.

1438

(62) Lopes, J. N. C.; Pádua, A. A. H. Molecular Force Field for Ionic 1439 Liquids Composed of Triflate or Bistriflylimide Anions. J. Phys. Chem. 1440 B 2004, 108, 16893-16898.

1441

(63) Gouveia, A. S. L.; Bernardes, C. E. S.; Tomé, L. C.; Lozinskaya, 1442 E. I.; Vygodskii, Y. S.; Shaplov, A. S.; Lopes, J. N. C.; Marrucho, I. M. 1443 Ionic Liquids with Anions Based on Fluorosulfonyl Derivatives: From 1444 Asymmetrical Substitutions to a Consistent Force Field Model. Phys. 1445 Chem. Chem. Phys. 2017, 19, 29617-29624.

1446

(64) Shimizu, K.; Almantariotis, D.; Gomes, M. F. C.; Pádua, A. A. 1447 H.; Canongia Lopes, J. N. Molecular Force Field for Ionic Liquids V: 1448 Hydroxyethylimidazolium, Dimethoxy-2- Methylimidazolium, and 1449 Fluoroalkylimidazolium Cations and Bis(Fluorosulfonyl)Amide, Per- 1450 fluoroalkanesulfonylamide, and Fluoroalkylfluorophosphate Anions. J. 1451 Phys. Chem. B 2010, 114, 3592-3600.

1452

(65) Berendsen, H. J. C.; Grigera, J. R.; Straatsma, T. P. The Missing 1453 Term in Effective Pair Potentials. J. Phys. Chem. A 1987, 91, 6269- 1454 6271.

1455

(66) Dang, L. X. Development of Nonadditive Intermolecular 1456 Potentials Using Molecular Dynamics: Solvation of $\mathrm{Li}+$ and F- Ions in 1457 Polarizable Water. J. Chem. Phys. 1992, 96, 6970-6977. 1458

(67) Martínez, L.; Andrade, R.; Birgin, E. G.; Martínez, J. M. 1459 PACKMOL: A Package for Building Initial Configurations for 1460 Molecular Dynamics Simulations. J. Comput. Chem. 2009, 30, 1461 2157-2164.

1462

(68) Bussi, G.; Donadio, D.; Parrinello, M. Canonical Sampling 1463 through Velocity Rescaling. J. Chem. Phys. 2007, 126, No. 014101. 1464

(69) Parrinello, M.; Rahman, A. Polymorphic Transitions in Single 1465 Crystals: A New Molecular Dynamics Method. J. Appl. Phys. 1981, 52, 1466 7182-7190.

1467

(70) Darden, T.; York, D.; Pedersen, L. Particle Mesh Ewald: An N. 1468 $\log (\mathrm{N})$ Method for Ewald Sums in Large Systems. J. Chem. Phys. 1469 1993, 98, 10089-10092.

1470

(71) Essmann, U.; Perera, L.; Berkowitz, M. L.; Darden, T.; Lee, H.; 1471 Pedersen, L. G. A Smooth Particle Mesh Ewald Method. J. Chem. 1472 Phys. 1995, 103, 8577-8593.

1473

(72) Humphrey, W.; Dalke, A.; Schulten, K. VMD: Visual Molecular 1474 Dynamics. J. Mol. Graphics 1996, 14, 33-38. 1475

(73) Brehm, M.; Kirchner, B. TRAVIS - A Free Analyzer and 1476 Visualizer for Monte Carlo and Molecular Dynamics Trajectories. J. 1477 Chem. Inf. Model. 2011, 51, 2007-2023. 
1479 (74) Hollóczki, O.; Macchiagodena, M.; Weber, H.; Thomas, M.; 1480 Brehm, M.; Stark, A.; Russina, O.; Triolo, A.; Kirchner, B. Triphilic 1481 Ionic-Liquid Mixtures: Fluorinated and Non-Fluorinated Aprotic 1482 Ionic-Liquid Mixtures. ChemPhysChem 2015, 16, 3325-3333.

1483 (75) Brehm, M.; Thomas, M.; Gehrke, S.; Kirchner, B. TRAVIS-A 1484 Free Analyzer for Trajectories from Molecular Simulation. J. Chem. 1485 Phys. 2020, 152, No. 164105.

1486 (76) Ozkanlar, A.; Clark, A. E. ChemNetworks: A Complex Network 1487 Analysis Tool for Chemical Systems. J. Comput. Chem. 2014, 35, 1488 495-505.

1489 (77) Vogel, H. The Law of the Relation between the Viscosity of 1490 Liquids and the Temperature. Phys. Z. 1921, 22, 645.

1491 (78) Fulcher, G. S. Analysis Of Recent Measurements Of The 1492 Viscosity Of Glasses. J. Am. Ceram. Soc. 1925, 8, 789-794.

1493 (79) Tammann, G.; Hesse, W. Die Abhängigkeit Der Viscosität von 1494 Der Temperatur Bie Unterkühlten Flüssigkeiten. Z. Anorg. Allg. Chem. 1495 1926, 156, 245-257.

1496 (80) Li, Z.; Bouchal, R.; Mendez-Morales, T.; Rollet, A. L.; Rizzi, C.; 1497 Le Vot, S.; Favier, F.; Rotenberg, B.; Borodin, O.; Fontaine, O.; 1498 Salanne, M. Transport Properties of Li-TFSI Water-in-Salt Electro1499 lytes. J. Phys. Chem. B 2019, 123, 10514-10521.

1500 (81) Marinaro, M.; Bresser, D.; Beyer, E.; Faguy, P.; Hosoi, K.; Li, 1501 H.; Sakovica, J.; Amine, K.; Wohlfahrt-Mehrens, M.; Passerini, S. 1502 Bringing Forward the Development of Battery Cells for Automotive 1503 Applications: Perspective of R\&D Activities in China, Japan, the EU 1504 and the USA. J. Power Sources 2020, 459, No. 228073.

1505 (82) Duboué-Dijon, E.; Laage, D. Characterization of the Local 1506 Structure in Liquid Water by Various Order Parameters. J. Phys. 1507 Chem. B 2015, 119, 8406-8418.

1508 (83) Russina, O.; Lo Celso, F.; Di Michiel, M.; Passerini, S.; 1509 Appetecchi, G. B.; Castiglione, F.; Mele, A.; Caminiti, R.; Triolo, A. 1510 Mesoscopic Structural Organization in Triphilic Room Temperature 1511 Ionic Liquids. Faraday Discuss. 2014, 167, 499.

1512 (84) Lo Celso, F.; Yoshida, Y.; Castiglione, F.; Ferro, M.; Mele, A.; 1513 Jafta, C. J.; Triolo, A.; Russina, O. Direct Experimental Observation of 1514 Mesoscopic Fluorous Domains in Fluorinated Room Temperature 1515 Ionic Liquids. Phys. Chem. Chem. Phys. 2017, 19, 13101-13110.

1516 (85) Russina, O.; Lo Celso, F.; Plechkova, N.; Jafta, C. J.; 1517 Appetecchi, G. B.; Triolo, A. Mesoscopic Organization in Ionic 1518 Liquids. Top. Curr. Chem. 2017, 375, 247-263.

1519 (86) Seki, T.; Chiang, K. Y.; Yu, C. C.; Yu, X.; Okuno, M.; Hunger, 1520 J.; Nagata, Y.; Bonn, M. The Bending Mode of Water: A Powerful 1521 Probe for Hydrogen Bond Structure of Aqueous Systems. J. Phys. 1522 Chem. Lett. 2020, 11, 8459-8469.

1523 (87) Brubach, J. B.; Mermet, A.; Filabozzi, A.; Gerschel, A.; Lairez, 1524 D.; Krafft, M. P.; Roy, P. Dependence of Water Dynamics upon 1525 Confinement Size. J. Phys. Chem. B 2001, 105, 430-435.

1526 (88) Cringus, D.; Jansen, T. L. C.; Pshenichnikov, M. S.; Wiersma, 1527 D. A. Ultrafast Anisotropy Dynamics of Water Molecules Dissolved in 1528 Acetonitrile. J. Chem. Phys. 2007, 127, No. 084507.

1529 (89) Yu, C. C.; Chiang, K. Y.; Okuno, M.; Seki, T.; Ohto, T.; Yu, X.; 1530 Korepanov, V.; Hamaguchi, Ho.; Bonn, M.; Hunger, J.; Nagata, Y. 1531 Vibrational Couplings and Energy Transfer Pathways of Water's 1532 Bending Mode. Nat. Commun. 2020, 11, No. 5977. 